\title{
Spin-orbit coupled particle in a spin bath
}

\author{
Peter Stano, ${ }^{1,2}$ Jaroslav Fabian, ${ }^{3}$ and Igor Žutić ${ }^{4}$ \\ ${ }^{1}$ Institute of Physics, Slovak Academy of Sciences, 84511 Bratislava, Slovakia \\ ${ }^{2}$ Department of Physics, Klingelbergstrasse 82, University of Basel, Switzerland \\ ${ }^{3}$ Institute for Theoretical Physics, University of Regensburg, D-93040 Regensburg, Germany \\ ${ }^{4}$ Department of Physics, University at Buffalo, New York 14260-1500, USA
}

(Received 28 August 2012; published 12 April 2013)

\begin{abstract}
We consider a spin-orbit coupled particle confined in a quantum dot in a bath of impurity spins. We investigate the consequences of spin-orbit coupling on the interactions that the particle mediates in the spin bath. We show that in the presence of spin-orbit coupling, the impurity-impurity interactions are no longer spin conserving. We quantify the degree of this symmetry breaking and show how it relates to the spin-orbit coupling strength. We identify several ways how the impurity ensemble can in this way relax its spin by coupling to phonons. A typical resulting relaxation rate for a self-assembled Mn-doped ZnTe quantum dot populated by a hole is $1 \mu \mathrm{s}$. We also show that decoherence arising from nuclear spins in lateral quantum dots is still removable by a spin echo protocol, even if the confined electron is spin-orbit coupled.
\end{abstract}

DOI: 10.1103/PhysRevB.87.165303

PACS number(s): 75.30.Et, 76.60.-k, 71.38.-k, 73.21.La

\section{INTRODUCTION}

A singly occupied quantum dot is the state of the art of a controllable quantum system in a semiconductor. ${ }^{1,2}$ Coherent manipulation of the particle spin has been demonstrated in lateral dots, where top gates allow for an astonishing degree of control by electric fields ${ }^{3-6}$ and in self-assembled dots, where a weaker control over the dot shape and position is compensated by the speed of the optical manipulation. ${ }^{7}$ In both of these major groups, there are two main spin-dependent interactions of the confined particle and the semiconductor environment: spin-orbit coupling embedded in the band structure, and spin impurities, which are either nuclear spins or magnetic atoms. ${ }^{8,9}$

A particle couples to an impurity spin dominantly through an exchange interaction, which conserves the total spin of the pair. ${ }^{10}$ This way, the electron spin in a lateral GaAs dot will decohere within $10 \mathrm{~ns}$ due to the presence of nuclei. ${ }^{11-17}$ Typically, such decoherence is considered a nuisance that can be partially removed by spin echo techniques prolonging the coherence to hundreds of microseconds. ${ }^{18-20}$ Whether that decoherence time can be extended further, e.g., by polarizing the impurities, ${ }^{21,22}$ is not clear, as the experimentally achieved degree of polarization has been so far insufficient, despite great efforts. ${ }^{23-25}$ On the other hand, a strong particle-impurity interaction is desired in magnetically doped quantum dots. ${ }^{26-42}$ Here, the confined particle is central for both supporting energetically, and assisting in creation, the desired magnetic order of the impurity ensemble. In fact, similar magnetic ordering can be traced to the studies of magnetic polarons in bulk semiconductors, for over 50 years. ${ }^{43}$ The formation of a magnetic polaron can be viewed as a cloud of localized impurity spins, aligned through exchange interaction with a confined carrier spin. ${ }^{4-47}$

The conservation of the spin by the impurity-particle interaction is a crucial property. For example, the spin relaxation of the impurity ensemble is impossible with only spin-conserving interactions at hand. This motivates us to consider possibilities to break this symmetry. The first and obvious candidate is the spin-orbit coupling (SOC). ${ }^{8,9}$ Despite being weak on the scale of the particle orbital energies, it dominates the relaxation of the particle spin in electronic dots, as is well known, ${ }^{48}$ because it is the dominant spin-nonconserving interaction. An additional motivation to further consider the implications of spin-orbit coupling comes from experiments in (II,Mn)VI quantum wells. The spin-orbit coupling in these structures can be responsible for an effective impurity-particle (Mn-hole) interaction $^{49,50}$ and lead to measured time scales $\sim 100 \mathrm{ps},{ }^{51}$ characteristic also for the magnetic polaron formation observed in (II,Mn)VI quantum dots. ${ }^{26,33,34}$

The questions we pose and answer in this work are as follows: Assuming the particle is weakly spin-orbit coupled, how strong are the effective spin-nonconserving interactions which appear in the impurity ensemble and what is their form? Is the induced particle decoherence still removable by spin echo? Is the particle efficient in inducing impurity ensemble spin relaxation, thereby limiting the achievable degree of the dynamical nuclear spin polarization $?^{52}$ Can the magnetic order be created through the spin-nonconserving particle mediated interactions, that is, is this a relevant magnetic polaron formation channel?

To address these questions, we develop here a framework allowing us to treat different particles and impurity spins in a unified manner. We apply our method to two specific systems: a lateral quantum dot in GaAs occupied by a conduction electron with nuclear spins of constituent atoms as the spin impurities, and a self-assembled ZnTe quantum dot occupied by a heavy hole doped with $\mathrm{Mn}$ atoms as the spin impurities (readily incorporated as $\mathrm{Mn}$ is isovalent with group-II atom $\mathrm{Zn}$ ). Both of these systems are quasi-two-dimensional, the particle spin-orbit coupling is weak compared to the particle orbital level spacing, and the particle-impurity interaction is weak compared to the particle orbital and spin level spacings. 2,34,53,54 As it is known, ${ }^{55}$ in this regime one can derive an effective Hamiltonian for the impurity ensemble only, in which the particle does not appear explicitly. This can be done including the particle-bath interaction perturbatively in the lowest order (see the scheme in Fig. 1). Our contribution is in showing how the procedure generalizes to a spin-orbit coupled particle. In addition, we use the resulting Hamiltonian for the calculation 
(a)

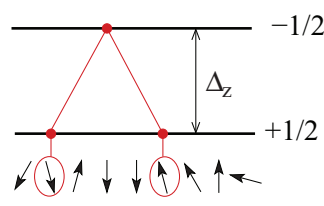

E 4

(b)

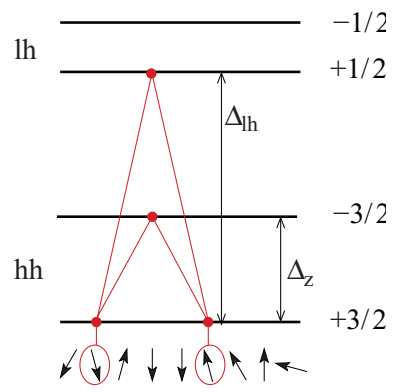

FIG. 1. (Color online) Effective interaction between impurities (encircled in red/gray) mediated by a confined particle (red/gray lines). (a) Electron is excited from the ground state of spin $+\frac{1}{2}$ into the closest spin- $\frac{1}{2}$ state (up by the Zeeman energy $\Delta_{z}$ ) upon flipping one of the impurities and deexcited back upon flipping another one. (b) Hole spectrum comprises heavy-hole- (hh) and light-hole- (lh) like states, the latter displaced by light-heavy-hole splitting $\Delta_{l h}$.

of the spin relaxation of the impurities which is phonon assisted (required to dissipate energy) and particle mediated (required to dissipate spin). We come up with (and evaluate the corresponding rates for) five possible mechanisms as to how the spin flips can proceed: shifts of the particle by the phonon electric field (Sec. IV B), position shifts of the impurity atoms (Sec. IV B), relative shifts of bulk bands (Sec. IV C), renormalization of the spin-orbit interactions due to band shifts (Sec. IV D), and spin-orbit interactions arising from the phonon electric field (Sec. IV E).

Our main findings are the following: (i) The spinnonconserving interactions couplings are proportional to the spin-conserving ones multiplied by some power of small parameter(s) which quantify the spin-orbit interaction. For the electron, the small parameter is the dot dimension divided by the spin-orbit length and the proportionality is linear. For the hole, the small parameters are the amplitudes of the light-hole admixtures into the heavy-hole states. The proportionality differs (from linear to quadratic) depending on which hole excited state mediates the interaction. The interaction form is given in Eq. (43), our main result. (ii) For the electron, the additional decoherence is removed by the spin echo, while for holes only a partial removal is possible. The latter is because, unlike for electrons, the spin-nonconserving coupling is mediated rather efficiently through higher excited states. (iii) The piezoelectric acoustic phonons are most efficient in relaxing the impurity spin. The resulting relaxation time is unobservably long for nuclear spins, while the hole-induced Mn spin relaxation time of $1 \mu \mathrm{s}$ is typical for a $10-\mathrm{nm}$ self-assembled quantum dot, where experimentally measured times for the polaron formation range from nanoseconds to subnanoseconds. ${ }^{26,33,34}$ From this we conclude that the interplay of spin-orbit coupling and phonons does not govern the dynamics of magnetic polaron formation at moderate Mn densities (few percent), but rather represents the spin-lattice relaxation time scale, similarly as is the case in quantum wells. $^{43,56}$ The analytical formulas derived in this work allow us to identify regimes (in addition to a very low Mn doping) where the particle-mediated spin relaxation could be relevant for the polaron formation: an example is a hole located at a

charged impurity. Furthermore, even when it is not the relevant time scale for magnetic polaron formation, the spin-lattice relaxation is an important quantity that determines the time scale on which the magnetization can be switched. Experimentally, time-resolved photoluminescence measurements of pulsed photoexcited carriers allow for a direct extraction of spin-lattice relaxation times in magnetic quantum dots. ${ }^{57}$ The use of our analytical findings therefore can help elucidate other trends in magnetization dynamics of quantum dots, not limited to magnetic polaron formation. ${ }^{57,58}$

The paper is organized as follows: In Sec. II, we introduce the description of the particle, focusing on the spin-orbit coupling. In Sec. III, we specify the particle-impurity interaction, define its important characteristics, and derive the effective Hamiltonian for the impurity ensemble. In Sec. IV, we calculate the spin relaxation rates for the impurity ensemble, after which we conclude and provide possible future directions including multiparticle effects. We put numerous technical details into the Appendices, with which the text is selfcontained.

\section{QUANTUM DOT STATES AND SPIN-ORBIT INTERACTION}

\section{A. Electron states}

In the single-band effective mass approximation, that we adopt, the Hamiltonian of a quantum dot electron is

$$
H_{\mathrm{dot}}=\frac{\mathbf{p}^{2}}{2 m}+V(\mathbf{r})+\frac{p_{z}^{2}}{2 m}+V_{z}(z)+g \mu_{B} \mathbf{B} \cdot \mathbf{J}+H_{\mathrm{SO}} .
$$

The underlying band structure is taken into account as a renormalization of the mass $m$ and the $g$ factor in the electron kinetic and Zeeman energies, respectively. The latter couples the external magnetic field $\mathbf{B}$, applied along a unit vector $\mathbf{s}_{0}$, to the electron spin through the vector of Pauli matrices $\sigma=2 \mathbf{J}$. Following, we assume a sizable (above $100 \mathrm{mT}$ ) external magnetic field in the electronic case, typically employed in experiments for the electron spin measurement ${ }^{48,59}$ and to slow down the impurity dynamics and the resulting decoherence. ${ }^{16}$ We neglect the orbital effects of this field, which is justified if the confinement length is much smaller than the magnetic length $l_{B}=\sqrt{ } 2 \hbar / e B$, where $-e$ is the electron charge. If the field is strong (above 1-2 T), the orbital effects important here are fully incorporated by a renormalization of the confinement length $l^{-4} \rightarrow l^{-4}+l_{B}^{-4}$.

The quantum dot is defined by the confinement potential $V+V_{z}$, which we separated into the in-plane and perpendicular contributions. The corresponding in-plane and perpendicular position and momentum components read as $\mathbf{R}=\{\mathbf{r}, z\}$ and $\mathbf{P}=\left\{\mathbf{p}, p_{z}\right\}$, respectively. Whenever we need an explicit form of the wave function, we assume, for convenience, a parabolic in-plane and a hard-wall perpendicular confinement:

$$
\begin{aligned}
V(\mathbf{r}) & =\frac{\hbar^{2}}{2 m l^{4}} r^{2} \equiv \frac{1}{2} m \omega^{2} r^{2}, \\
V_{z}(z) & = \begin{cases}0, & 0<z<w \\
\infty, & \text { otherwise. }\end{cases}
\end{aligned}
$$

The confinement lengths $l$ and $w$ characterize a typical extent of the wave function in the lateral and perpendicular directions, 
respectively. The confinement energy $\hbar \omega$ is a parameter alternative to $l$. We stress that our results do not rely on the specific confinement form in any way, as long as the dot is quasi-two-dimensional, a condition which for the adopted example reads as $l \gg w$. Typical values for lateral quantum dots in GaAs are $l=30 \mathrm{~nm}$ and $w=8 \mathrm{~nm}$.

The last term in Eq. (1) is the spin-orbit interaction ${ }^{8}$

$$
H_{\mathrm{SO}}=\frac{\hbar}{2 m l_{\mathrm{d}}}\left(-\sigma_{x} p_{x}+\sigma_{y} p_{y}\right)+\frac{\hbar}{2 m l_{\mathrm{br}}}\left(\sigma_{x} p_{y}-\sigma_{y} p_{x}\right) \text {, }
$$

comprising the Dresselhaus term, which arises in zinc-blende structures grown along the [001] axis and the Bychkov-Rashba term, which is a consequence of the strong perpendicular confinement. The interactions are parametrized by the spinorbit lengths $l_{\mathrm{SO}} \in\left\{l_{\mathrm{d}}, l_{\mathrm{br}}\right\}$, typically a few microns in GaAs heterostructures.

Assume first that the spin-orbit coupling is absent. To be able to treat the electron and the hole (each referred to as the particle) on the same footing, we introduce the following notation:

$$
\left|\Psi_{p}\right\rangle=|J\rangle \otimes\left|\Phi_{a}^{J}\right\rangle \quad \text { (zero SOC). }
$$

The complete particle wave function, for which we use the Greek letter $\Psi$, is a two- (electron) or four- (hole) component spatially dependent spinor. Its label $p=\{J, a\}$ indicates that the wave function is separable into a (position-independent) spinor $|J\rangle$ and a scalar position-dependent complex amplitude $|\Phi\rangle$. The former is labeled by the particle angular momentum in units of $\hbar, J= \pm \frac{1}{2}$ (electron), $J= \pm \frac{3}{2}, \pm \frac{1}{2}$ (hole; alternatively, we use hh for $\frac{3}{2}$ and $\mathrm{lh}$ for $\frac{1}{2}$ labels). The set of orbital quantum numbers $a$ depends on the confinement potential. For the choice in Eqs. (2), it is a set of three numbers $a=\{n m, k\}$, with $n$ the main and $m$ the orbital quantum number $(\bar{m} \equiv-m)$ of a Fock-Darwin state, and $k$ the label of the subband in the perpendicular hard-wall confinement. Finally, for the particle ground state, we omit the index $a$, or use $G \equiv\{J, 00,0\}$ in place of $p$. The electron ground state is thus denoted by

$$
\left|\Psi_{1 / 2}\right\rangle=|1 / 2\rangle \otimes\left|\Phi_{G}\right\rangle,
$$

where the direction of the spin-up spinor $|1 / 2\rangle$ is set by the external field along $\mathbf{s}_{0}$.

Let us now consider the spin-orbit coupling. It turns out that for electrons the spin-orbit effects on the wave function can be in the leading order written as ${ }^{60,61}$

$$
\left|\Psi_{p}\right\rangle=U|J\rangle \otimes\left|\Phi_{a}^{J}\right\rangle .
$$

Here, $U$ is a unitary $2 \times 2$ matrix of a spinor rotation

$$
U(\mathbf{r})=\exp \left[-\mathrm{i} \mathbf{n}_{\mathrm{SO}}(\mathbf{r}) \cdot \mathbf{J}\right],
$$

parametrized by an in-plane position-dependent vector

$$
\mathbf{n}_{\mathrm{SO}}(\mathbf{r})=-\left(\frac{x}{l_{\mathrm{d}}}-\frac{y}{l_{\mathrm{br}}}, \frac{x}{l_{\mathrm{br}}}-\frac{y}{l_{\mathrm{d}}}, 0\right) .
$$

A weak spin-orbit coupling allows us to label states with the same quantum numbers as for no spin-orbit case, as there is a clear one-to-one correspondence. The enormous simplification, that the unitary matrix in Eq. (6) does not depend on the quantum numbers $p$, is due to the special form of the spin-orbit coupling in Eq. (3), which has several interesting consequences. ${ }^{62-65}$ To calculate the spin relaxation or spinorbit-induced energy shifts, on which $U$ has no effects, one has to go beyond the leading order given in Eq. (6). ${ }^{66}$ However, we will see that here $U$ will result in spin-nonconserving interactions, and it is thus enough to consider the leading order. For the same reason, we neglected the cubic Dresselhaus term in Eq. (3), which is here, unlike usually, ${ }^{67}$ an excellent approximation.

\section{B. Hole states}

For holes, we restrict to the four-dimensional subspace of the light- and heavy-hole subbands. Neglecting the spin-orbit coupling, they correspond to the angular momentum states $J= \pm \frac{3}{2}$, and $\pm \frac{1}{2}$, respectively. We use the confinement potential in Eqs. (2), setting the confinement energy in the heavy-hole subband to $20 \mathrm{meV}$, which gives $l \approx 4 \mathrm{~nm}$ and the light-heavy-hole splitting to $\Delta_{\mathrm{lh}}=100 \mathrm{meV}$, which gives $w \approx 2 \mathrm{~nm}$. The atomic spin-orbit coupling manifests itself as the orbital splitting of the light and heavy holes from the spin-orbit split-off band (which is energetically far from the states considered in this paper), and as a coupling of the light and heavy holes at finite momenta. The latter effect we refer to as the hole spin-orbit coupling. As the nomenclature concerning this coupling varies in literature, we stress that for holes, we do not consider higher-order effects, which give rise to spin-orbit interactions similar in form to the electronic Dresselhaus and Rashba terms given in Eq. (3): such higher-order terms admixing heavy-hole states do not induce additional interactions within the impurity ensemble, whereas, as we show in Appendix F, the electric field (interface) induced spin-orbit coupling leads to effects analogous but smaller than those we consider. Within this model, we derive the spin-orbit coupled wave functions from the corresponding $4 \times 4$ sector of the Kohn-Luttinger Hamiltonian in Appendix A, and get the hole ground state as

$$
\begin{aligned}
\left|\Psi_{3 / 2}\right\rangle= & |3 / 2\rangle \otimes\left|\Phi_{00,0}^{\mathrm{hh}}\right\rangle+\lambda_{1}|1 / 2\rangle \otimes\left|\Phi_{01,1}^{\mathrm{lh}}\right\rangle \\
& +\lambda_{0}|-1 / 2\rangle \otimes\left|\Phi_{02,0}^{\mathrm{lh}}\right\rangle .
\end{aligned}
$$

We used the notation explained below Eq. (4). The FockDarwin states in the heavy-hole (hh) and light-hole (lh) subbands differ, due to different effective masses. The key quantities are the scalars $\lambda$, which quantify the light-heavyhole mixing. The spin-nonconserving interactions, as well as the resulting spin relaxation rates, will scale with these scalars. For our parameters, which we list for convenience in Appendix $\mathrm{E}$, we get $\lambda_{0} \approx \lambda_{1} \approx 0.05$. In addition to the ground state, we will need also the lowest excited state in the heavy-hole subband, which is the time-reversed copy of Eq. (9):

$$
\begin{aligned}
\left|\Psi_{-3 / 2}\right\rangle= & |-3 / 2\rangle \otimes\left|\Phi_{00,0}^{\mathrm{hh}}\right\rangle-\lambda_{1}|-1 / 2\rangle \otimes\left|\Phi_{0 \overline{1}, 1}^{\mathrm{lh}}\right\rangle \\
& +\lambda_{0}|1 / 2\rangle \otimes\left|\Phi_{0 \overline{2}, 0}^{\mathrm{hh}}\right\rangle,
\end{aligned}
$$

and also the lowest state in the light-hole subband

$$
\begin{aligned}
\left|\Psi_{1 / 2}\right\rangle= & |1 / 2\rangle \otimes\left|\Phi_{00,0}^{\mathrm{lh}}\right\rangle+\lambda_{1}^{\prime}|3 / 2\rangle \otimes\left|\Phi_{0 \overline{1}, 1}^{\mathrm{hh}}\right\rangle \\
& -\lambda_{0}^{\prime}|-3 / 2\rangle \otimes\left|\Phi_{0 \overline{2}, 0}^{\mathrm{lh}}\right\rangle,
\end{aligned}
$$


which is surprisingly effective in inducing the spinnonconserving coupling among impurities, as we will see. We make a few notes here: First, in the spherical approximation that we adopt, the Kohn-Luttinger Hamiltonian conserves the angular momentum, so that all components in each of Eqs. (9)-(11) have the same value of $J+m$. Second, the mixing is stronger in the light-hole subband $\lambda_{1}^{\prime} \approx 0.15$ and $\lambda_{0}^{\prime} \approx 0.11$. This is because the admixing states are closer in energy: the Fock-Darwin excitation energies add to and subtract from the light-heavy-hole splittings in the heavyand light-hole cases, respectively, as evidenced by Eqs. (A9) and (A11). Third, we will be interested in the case of zero external magnetic field for holes. Unlike for electrons, such a field is here not required to split the two states in Eqs. (9) and (10), as the splitting arises due to the spin impurities. As we will see, this splitting will be of the order of few meV. Compared to this, the hole Zeeman energy is negligible up to fields of several Tesla. In addition, the external field suppresses an interesting feedback between the particle and impurities. ${ }^{68}$ Finally, we note that one could relate the first-order and unperturbed hole states analogously to the electron case, introducing a unitary transformation $U$, whose matrix elements are the coefficients appearing in Eqs. (9)-(11). However, since here the transformation does not have any appealing form similar to the one in Eq. (7), we do not explicitly construct the matrix $U$ for holes.

\section{EFFECTIVE HAMILTONIAN}

In this section, we introduce the particle-impurity exchange interaction, in a unified description for both electrons and holes. The interaction manifests itself as the Knight field acting on the impurities and the Overhauser field acting on the particle. (The fields are defined as the exchange-interaction expectation value in the corresponding subsystem state.) Historically, the terminology was initially applied to nuclei and here we also use it for Mn spins. With the help of these fields, we define the unperturbed basis of the particleimpurity system, for which we derive the effective interaction Hamiltonian treating the nondiagonal exchange terms perturbatively. Finally, we define the spin-conserving versus spin-nonconserving interaction terms and analyze their relative strength.

Our strategy can be viewed also in the following alternative way. To derive the spin-orbit coupling effects on the effective impurity interactions, we proceed in two steps: First, we unitarily transform the particle basis to remove the spin-orbit coupling in the lowest order. The spin-orbit coupled basis transformation renormalizes the particle-impurity exchange interaction and breaks its spin-rotational symmetry. Then, we integrate out the particle degrees of freedom by a second unitary transformation, using the Löwdin (equivalently here, the Schrieffer-Wolff) transformation, which leaves us with effective interactions concerning impurities only.

\section{A. Particle-impurity interaction}

The particle interacts with impurities by the Fermi contact interaction $^{52,69}$

$$
H_{F}=\sum_{n} H_{F}^{n}=-\sum_{n} \beta \delta\left(\mathbf{R}-\mathbf{R}_{n}\right) \mathbf{J} \cdot \mathbf{I}^{n} .
$$

Here, $n=1,2 \ldots$ labels the impurities located at positions $\mathbf{R}_{n}=\left(\mathbf{r}_{n}, z_{n}\right)$ with corresponding spin operators $\mathbf{I}^{n}$ in units of $\hbar$. The impurities have spin $I$ and density $1 / v_{0}$. For impurities with different magnetic moments (as for nuclei of different elements), the coupling $\beta$ should have the index $n$, but we will not consider this minor complication. Even though the particle wave function formally extends to infinity, one can define the number of impurities with which the particle interacts appreciably as $N=V / v_{0}$, with the dot volume $V$ given by ${ }^{13}$

$$
1 / V=\int d^{3} \mathbf{R}\left|\Phi_{G}(\mathbf{R})\right|^{4}
$$

The maximal value of the Hamiltonian $H_{F}$, if all impurities are aligned with the particle, is

$$
E=-\sum_{n} \beta\left|\Phi_{G}\left(\mathbf{R}_{n}\right)\right|^{2} J I=-\beta J I / v_{0} .
$$

The impurity Zeeman energy is

$$
H_{n Z}=\sum_{n} g_{n} \mu_{\mathrm{imp}} \mathbf{I}^{n} \cdot \mathbf{B},
$$

with $g_{n}$ the impurity $g$ factor. For GaAs lateral dots, we consider nuclear spins as impurities, with $I=\frac{3}{2}$ and $\mu_{\text {imp }}$ the nuclear magneton $\mu_{N}$, and $1 / v_{0}$ is the GaAs atomic density. For ZnTe dots, the impurities are intentionally doped Mn atoms, with $I=\frac{5}{2}$ and $\mu_{\mathrm{imp}}$ the Bohr magneton $\mu_{B}$, and the impurity density is parametrized by $x_{\mathrm{Mn}}$, the fraction of cations replaced by $\mathrm{Mn}$ atoms, typically $x_{\mathrm{Mn}}=1 \%$.

\section{B. Knight field}

Assume the particle sits in the ground state $G$. In the lowest order of the the particle-impurity interaction, a particular impurity spin couples to a local field, called the Knight field. We define it in units of energy by writing

$$
\mathbf{K}^{n} \cdot \mathbf{I}^{n} \equiv\left\langle\Psi_{G}\left|H_{F}^{n}\right| \Psi_{G}\right\rangle,
$$

from which, using Eq. (12), we get

$$
\mathbf{K}^{n}=-\beta\left\langle\Psi_{G}\left|\delta\left(\mathbf{R}-\mathbf{R}_{n}\right) \mathbf{J}\right| \Psi_{G}\right\rangle .
$$

Using Eq. (6), the Knight field of an electron is

$$
\begin{aligned}
\mathbf{K}^{n} & =-\beta\left|\Phi_{G}\left(\mathbf{R}_{n}\right)\right|^{2}\left\langle 1 / 2\left|U^{\dagger}\left(\mathbf{R}_{n}\right) \mathbf{J} U\left(\mathbf{R}_{n}\right)\right| 1 / 2\right\rangle \\
& =-\beta(1 / 2)\left|\Phi_{G}\left(\mathbf{R}_{n}\right)\right|^{2}\left\langle\mathbf{s}\left(\mathbf{R}_{n}\right)\right\rangle .
\end{aligned}
$$

It points along the direction of the electron spin at the position of the $n$th impurity, introduced as a unit vector $\left\langle\mathbf{s}\left(\mathbf{R}_{n}\right)\right\rangle \equiv R_{U\left(\mathbf{R}_{n}\right)}\left[\mathbf{s}_{0}\right]$. The operator $R_{U}$ is defined such that it performs the same rotation on vectors, as $U$ does on spinors. The explicit form of $R$ is the one in Eq. (7), if generators of rotations in three dimensions are used, $\left(J_{k}\right)_{l m}=$ $-\mathrm{i} \epsilon_{k l m}$. As is apparent from Eq. (18), evaluating the Knight field with perturbed electron wave functions is equivalent to evaluating a unitarily transformed interaction $H_{F} \rightarrow U^{\dagger} H_{F} U$ with unperturbed electron wave functions.

We get the Knight field of the hole as (see Appendix B)

$$
\left[K_{x}^{n}, K_{y}^{n}, K_{z}^{n}\right]=-\beta\left[\lambda_{1} \operatorname{Re} f_{n}, \lambda_{1} \operatorname{Im} f_{n}, 3\left|\Phi_{00,0}^{h h}\left(\mathbf{R}_{n}\right)\right|^{2} / 2\right],
$$

where we abbreviated $f_{n}=\sqrt{3} \Phi_{01,1}^{\mathrm{lh}}\left(\mathbf{R}_{n}\right) \Phi_{00,0}^{\mathrm{hh} *}\left(\mathbf{R}_{n}\right)$ and neglected contributions of higher orders in $\lambda$. Rather than the 
exact form, we note that without the spin-orbit coupling, the direction of the Knight field is fixed globally (along the external magnetic field for electrons, $\mathbf{s}_{0}=\mathbf{B} / B$ and along the $\mathrm{z}$ axis, the spin direction of heavy holes, for holes $\mathbf{S}_{0}=\hat{\mathbf{z}}$ ). The spin-orbit interaction deflects the Knight field in a position-dependent way, the deflection being in the leading order linear in the small parameter characterizing the spin-orbit interaction. In this respect, Eqs. (18) and (19) are the same.

\section{Basis}

The total field aligning the impurity spin is the sum of the Knight field and the external field

$$
\mathcal{B}^{n}=\mathbf{K}^{n}+g_{n} \mu_{\text {imp }} \mathbf{B}
$$

The typical energy scale of the Knight field of an electron in a lateral dot is tens of peV, which corresponds to the impurity in an external field of $1 \mathrm{mT}$. For a hole in a self-assembled dot, the Knight field is of the order of $100 \mu \mathrm{eV}$, corresponding to the external field of $0.3 \mathrm{~T}$. Based on this, in the following analysis we mostly consider typical situations, in which the total field is dominated by the external field for nuclear spins (electronic case) and the Knight field for Mn spins (hole case).

We now introduce for each impurity a rotated (primed) coordinate system, in which the unit vector $\hat{\mathbf{z}}^{\prime}$ is along the total field $\mathcal{B}^{n}$. Formally, the rotation is performed by operator $R_{\mathcal{B}^{n}}$ defined by the relation between the unit vectors

$$
\hat{\mathbf{z}}^{\prime}=R_{\mathcal{B}^{n}}\left[\hat{\mathbf{s}}_{0}\right]
$$

The orientation of the in-plane axes $x^{\prime}, y^{\prime}$ in the plane perpendicular to $\hat{\mathbf{z}}^{\prime}$ is arbitrary, and we denote $\mathbf{r}_{ \pm}^{\prime}=\hat{\mathbf{x}}^{\prime} \pm \mathrm{i} \hat{\mathbf{y}}^{\prime}$. We define the impurity ensemble basis states as tensor products of states with a definite spin projection along the locally rotated axis $z^{\prime}$ :

$$
|\mathcal{I}\rangle=\left|I_{z^{\prime}}^{1}\right\rangle \otimes\left|I_{z^{\prime}}^{2}\right\rangle \otimes \cdots \otimes\left|I_{z^{\prime}}^{N}\right\rangle
$$

The spin projections take discrete values $I_{z^{\prime}}^{n} \in\{I, I-$ $1, \ldots,-I\}$. We use $\mathcal{I}$ as the collective index of the impurities. With this, we are now ready to introduce the complete system basis, as spanning the states

$$
\left|\Psi_{p}\right\rangle \otimes|\mathcal{I}\rangle \equiv\left|\Psi_{p}\right\rangle \otimes\left|I_{z^{\prime}}^{1}\right\rangle \otimes\left|I_{z^{\prime}}^{2}\right\rangle \otimes \cdots \otimes\left|I_{z^{\prime}}^{N}\right\rangle,
$$

with the corresponding energies

$$
E_{p, \mathcal{I}}=E_{p}+\sum_{n} E_{I_{z^{\prime}}^{n}}=E_{p}+\sum_{n} \mathcal{B}^{n} I_{z^{\prime}}^{n}
$$

comprising the particle energy and the Zeeman energies of impurities in the corresponding total fields.

\section{The substantial gap assumption: Overhauser field}

In addition to the Knight field, another consequence of the particle-impurity interaction from Eq. (12) is the effective field experienced by the particle spin, known as the Overhauser field $^{2}$ O. To express it in the energy units, it is helpful to consider the matrix elements of the particle-impurity interaction within the subspace of the lowest two electron states $J, J^{\prime} \in S \equiv\left\{\frac{1}{2},-\frac{1}{2}\right\}$ :

$$
\begin{aligned}
& \left\langle\Psi_{J}\left|-\beta \sum_{n} \delta\left(\mathbf{R}-\mathbf{R}_{n}\right) \mathbf{J} \cdot \mathbf{I}^{n}\right| \Psi_{J^{\prime}}\right\rangle \\
& =-\beta \sum_{n}\left|\Phi_{G}\left(\mathbf{R}_{n}\right)\right|^{2} R_{U_{n}^{\dagger}}\left[\mathbf{I}^{n}\right] \cdot\left\langle J|\mathbf{J}| J^{\prime}\right\rangle .
\end{aligned}
$$

We introduce the field $\mathbf{O}$ as

$$
\left.H_{F}\right|_{S} \equiv \mathbf{O} \cdot \mathbf{J}
$$

where the subscript $S$ refers to the subspace comprising a pair of time-reversed particle states and the Overhauser field depends on the choice of $S$. To quantify the Overhauser field, we give up on trying to track the microscopic state of the impurities and instead introduce the averaging (denoted by an overline) over impurity ensembles

$$
\overline{I_{a}^{n}}=0, \quad \overline{I_{a}^{n} I_{b}^{m}}=\delta_{n m} \delta_{a b} I(I+1) / 3,
$$

which characterize unpolarized and isotropic ensembles. Nuclear spins, unless intentionally polarized in dynamical nuclear polarization schemes, ${ }^{22,70,71}$ are usually well described by Eq. (27). The same holds for Mn spins initially, before the particle enters the dot and the polarization starts to build up.

Equation (27) gives a zero Overhauser field on average, but with a finite dispersion, quantifying a typical value. For electrons, we get the well-known result ${ }^{13,72}$

$$
\begin{aligned}
\overline{\mathbf{O}^{2}} & =\beta^{2} \sum_{n m}\left|\Phi_{G}\left(\mathbf{R}_{n}\right)\right|^{2}\left|\Phi_{G}\left(\mathbf{R}_{m}\right)\right|^{2} \overline{R_{U_{n}^{\dagger}}\left[\mathbf{I}^{n}\right] \cdot R_{U_{m}^{\dagger}}\left[\mathbf{I}^{m}\right]} \\
& =\beta^{2} \sum_{n}\left|\Phi_{G}\left(\mathbf{R}_{n}\right)\right|^{4} I(I+1)=I(I+1)\left(\beta / v_{0}\right)^{2} / N,
\end{aligned}
$$

stating that the typical value of the Overhauser field is inversely proportional to the square root of the number of impurity spins within the dot. The spin-orbit coupling, equivalent to a position-dependent spin coordinate frame rotation, does not influence the result at all, as Eq. (27) assumes isotropic noninteracting impurities. For our parameters, the typical Overhauser field value is $0.15 \mu \mathrm{eV}$, which corresponds to external field of $10 \mathrm{mT}$. The energy splitting of the electron spin opposite states is therefore for our case dominated by the Zeeman, rather than the Overhauser, field.

For holes, we will not write the Overhauser field explicitly as a vector. Instead, we calculate directly the typical matrix elements of the particle-impurity interaction within the heavyhole subspace with the spin-orbit renormalized wave functions. We leave details for Appendix B and state the results: the diagonal terms are

$$
\overline{\left|\left\langle\Psi_{ \pm 3 / 2}\left|H_{F}\right| \Psi_{ \pm 3 / 2}\right\rangle\right|^{2}} \approx(3 / 4) I(I+1)\left(\beta / v_{0}\right)^{2} / N,
$$

where we neglected small contributions of the spin-orbit coupling. An important difference to an analogous result for the electrons [Eq. (28)] is the energy scale. Here, the typical value for the diagonal Overhauser field is several $\mathrm{meV}$, which corresponds to huge external fields of many Tesla. The energy splitting of the hole is thus dominated by the Overhauser, rather 
than Zeeman, field. On the other hand, the off-diagonal element is nonzero only in the presence of the spin-orbit coupling

$$
\overline{\left|\left\langle\Psi_{-3 / 2}\left|H_{F}\right| \Psi_{3 / 2}\right\rangle\right|^{2}} \sim 2 I(I+1)\left(\lambda_{0} \beta / v_{0}\right)^{2} / N .
$$

The impurity spins may induce transition (precession) of the heavy-hole spin due to the transversal component of the Overhauser field, which is smaller by a factor of $\lambda_{0}$ compared to the diagonal component. For our parameters, the transversal component is of the order of tens of $\mu \mathrm{eV}$, so for the heavy-hole spin precession to occur, the two spin opposite states have to be degenerate with respect to this energy [which normally does not occur because of the diagonal term (28)].

Having compared the typical energy splittings of the particle induced by the effective Overhauser field, versus the external magnetic field, we are now ready to discuss the crucial assumption for the derivation which will follow. It is the assumption that the particle is fixed to its ground state by an energy gap, irrespective of the evolution of the impurity ensemble. This requires that spin flips of impurities cost much less in energy than the particle transitions

$$
\Delta E_{I^{n}} \ll \Delta E_{p} .
$$

For electrons, this assumption is guaranteed as both the particle and impurities spin-flip costs are dominated by the Zeeman energy, proportional to the magnetic moment, which is much larger for the electron than for a nuclear spin $\mu_{\text {imp }}=\mu_{N} \sim$ $10^{-3} \mu_{B}$. On the other hand, for holes for which the particle and impurity magnetic moments are comparable, the above condition is also fulfilled since the particle spin-flip energy cost is dominated by the Overhauser field.

\section{E. Effective Hamiltonian}

Once the particle is fixed to its ground state (the substantial gap assumption), the particle excited states can be integrated out perturbatively ${ }^{55,73,74}$ resulting in an effective Hamiltonian for the impurity ensemble $H_{\text {eff }}$. For this purpose, we split the interaction Hamiltonian to

$$
H_{F} \equiv H_{F}^{0}+H_{F}^{\prime},
$$

where the diagonal part

$$
H_{F}^{0}=\left\langle\Psi_{G}\left|H_{F}\right| \Psi_{G}\right\rangle=\sum_{n} \mathbf{K}^{n} \cdot \mathbf{I}^{n}
$$

together with the external field, defines the unperturbed Hamiltonian $H_{0}=H_{p}+H_{n Z}+H_{F}^{0}$ and the basis, so that

$$
\left\langle\Psi_{p} \otimes \mathcal{I}_{A}\left|H_{n Z}+H_{F}^{0}\right| \Psi_{q} \otimes \mathcal{I}_{B}\right\rangle \propto \delta_{p q} \delta_{A B},
$$

where $\mathcal{I}_{A}, \mathcal{I}_{B}$ denote arbitrary basis states of the impurity ensemble. We also note that

$$
\left\langle\Psi_{G} \otimes \mathcal{I}_{A}\left|H_{F}^{\prime}\right| \Psi_{G} \otimes \mathcal{I}_{B}\right\rangle=0 .
$$

Using Löwdin theory, ${ }^{75,76}$ the matrix elements of the effective Hamiltonian, in the lowest order in the nondiagonal part $H_{F}^{\prime}$, are

$$
\left\langle\mathcal{I}_{A}\left|H_{\mathrm{eff}}\right| \mathcal{I}_{B}\right\rangle=\left\langle\Psi_{G} \otimes \mathcal{I}_{A}\left|\quad H_{0}+\sum_{p \neq G, \mathcal{I}^{*}}\left(\frac{1 / 2}{E_{G \mathcal{I}_{A}}-E_{p \mathcal{I}^{*}}}+\frac{1 / 2}{E_{G \mathcal{I}_{B}}-E_{p \mathcal{I}^{*}}}\right) H_{F}^{\prime}\right| \Psi_{p} \otimes \mathcal{I}^{*}\right\rangle\left\langle\Psi_{p} \otimes \mathcal{I}^{*}\left|H_{F}^{\prime}\right| \Psi_{G} \otimes \mathcal{I}_{B}\right\rangle
$$

where the summation proceeds through the excited particle states and a complete basis of impurities. The substantial gap assumption assures that all states reachable by $H_{F}^{\prime}$ have the energy dominated by the particle, so that we can put $E_{G \mathcal{I}}-$ $E_{p \mathcal{I}^{*}} \approx E_{G}-E_{p}$. The summation over the impurities then gives an identity

$$
\begin{aligned}
& \left\langle\mathcal{I}_{A}\left|H_{\mathrm{eff}}\right| \mathcal{I}_{B}\right\rangle \\
& =\left\langle\Psi_{G} \otimes \mathcal{I}^{A}\left|H_{0}+H_{F}^{\prime} \sum_{p \neq G} \frac{\left|\Psi_{p}\right\rangle\left\langle\Psi_{p}\right|}{E_{G}-E_{p}} H_{F}^{\prime}\right| \Psi_{G} \otimes \mathcal{I}^{B}\right\rangle .
\end{aligned}
$$

Since the impurity states now only sandwich both sides of the equation, we can equate the operators

$$
H_{\mathrm{eff}}=\left\langle\Psi_{G}\left|H_{0}\right| \Psi_{G}\right\rangle+\sum_{p \neq G} \frac{\left\langle\Psi_{G}\left|H_{F}^{\prime}\right| \Psi_{p}\right\rangle\left\langle\Psi_{p}\left|H_{F}^{\prime}\right| \Psi_{G}\right\rangle}{E_{G}-E_{p}} .
$$

Even though this looks like the standard second-order perturbation theory result, note that even after taking matrix elements with respect to the particle states, the expressions still contain the quantum mechanical operators of the impurity spins. On the other hand, by taking the expectation value, the particle degrees of freedom disappear from the effective Hamiltonian. The first term is a sum of the particle ground-state energy and the impurities energy in the Knight field

$$
\left\langle\Psi_{G}\left|H_{0}\right| \Psi_{G}\right\rangle=E_{G}+\sum_{n} \mathcal{B}^{n} \cdot \mathbf{I}^{n} .
$$

To simplify the notation of the second term in Eq. (38), we introduce

$$
\left\langle\Psi_{G}\left|H_{F}^{\prime}\right| \Psi_{p}\right\rangle=\left\langle\Psi_{G}\left|H_{F}\right| \Psi_{p}\right\rangle \equiv \sum_{n} \mathbf{A}^{n} \cdot \mathbf{I}^{n},
$$

so that the $p$-state-dependent complex vector $\mathbf{A}$ is

$$
\mathbf{A}^{n}=-\beta\left\langle\Psi_{G}\left|\delta\left(\mathbf{R}-\mathbf{R}_{n}\right) \mathbf{J}\right| \Psi_{p}\right\rangle .
$$

We now transform vectors $\mathbf{A}$ and spin operators $\mathbf{I}$ into the coordinate system along the total field of each impurity

$$
\tilde{\mathbf{A}}^{n}=R_{\mathcal{B}^{n}}^{-1}\left[\mathbf{A}^{n}\right], \quad \tilde{\mathbf{I}}^{n}=R_{\mathcal{B}^{n}}^{-1}\left[\mathbf{I}^{n}\right] .
$$

The $z$ component of a rotated vector is its projection along the direction of the local total field, e.g., $\tilde{I}_{z}=\mathbf{I} \cdot \hat{\mathbf{z}}^{\prime}$ and similarly for A. Omitting the constant $E_{G}$, we rewrite Eq. (38) with the 
new notation and arrive at our main result [see Eq. (D1) for its component form]

$$
H_{\mathrm{eff}}=\sum_{n} \mathcal{B}^{n} \tilde{I}_{z}^{n}+\sum_{p \neq G} \sum_{n, m} \frac{1}{E_{G}-E_{p}}\left(\tilde{\mathbf{A}}^{n} \cdot \tilde{\mathbf{I}}^{n}\right)\left(\tilde{\mathbf{A}}^{m} \cdot \tilde{\mathbf{I}}^{m}\right)^{\dagger} .
$$

The first term defines the spin-flip energy cost and the spin quantization axis given in Eq. (21). The interactions described by the second term can be classified as spin conserving (spin nonconserving) according to rotated operator $\tilde{\mathbf{I}}$ components parallel (perpendicular) to a global axis $\hat{\mathbf{s}}_{0}$, as we will show in the following. To further demonstrate the usefulness and generality of Eq. (43), we show that known results follow as special limits, and how the consequences of the spin-orbit coupling on the impurities interactions can be drawn from the formula. We also note that the derivation would proceed in the same way even if $G$ were not the particle ground state. The only requirement for the validity of Eq. (43) is that the state $G$ is far enough in energy from other particle states so that Eq. (31) is valid. For example, thermal excitations of the particle would result in a thermal average of the effective Hamiltonian (the vectors $\mathbf{A}$ and energies $\mathcal{B}$ do depend on $G$ ). We do not pursue a finite-temperature regime further here, and assume the thermal energy $k_{B} T$ is small such that the particle stays in the ground state.

Before we evaluate vectors $\tilde{\mathbf{A}}$ in specific cases, we note an important property of the effective Hamiltonian. Namely, for both holes and electrons, the lowest excited state is much closer to the ground state (split by the Zeeman energy) compared to higher excited states (split by orbital excitation energies). If the mediated interactions are dominated by this low-lying excited state, we have

$$
H_{\mathrm{eff}}=\sum_{n} \mathcal{B}^{n} \tilde{I}_{z}^{n}+\sum_{n, m} \frac{1}{E_{G}-E_{p}}\left(\tilde{\mathbf{A}}_{G p}^{n} \cdot \tilde{\mathbf{I}}^{n}\right)\left(\tilde{\mathbf{A}}_{G p}^{m} \cdot \tilde{\mathbf{I}}^{m}\right)^{\dagger},
$$

where we made explicit the dependence of vectors $\mathbf{A}$ on the ground and excited states using them as indexes. We note that $A_{p p^{\prime}}=A_{T(p) T\left(p^{\prime}\right)}^{\dagger}$, up to a phase (which cancels in the $H_{\text {eff }}$ because of the Hermitian conjugated term), where $T(p)$ is the state index of a time reversal of state $p$. Choosing $p=$ $T(G)$ with $G$, first, spin-up, and second, spin-down state, we get that the effective Hamiltonians for the two choices differ only by the sign in the second term in Eq. (44). This crucial property, which results in the particle spin decoherence being to a large extent removable by the spin echo protocols, ${ }^{15,16}$ is thus preserved in the presence of the spin-orbit coupling: It follows as a general result whenever the spin opposite particle state is the virtual state most effective in mediating the impurity interactions.

\section{F. Effective Hamiltonian symmetry and magnitude of the spin-nonconserving interactions}

For electrons, we get from Eq. (18)

$$
\tilde{\mathbf{A}}^{n}=R_{\mathcal{B}^{n}}^{-1}\left[\mathbf{A}^{n}\right]=\epsilon_{p}^{n} R_{\mathcal{B}^{n}}^{-1} \circ R_{U_{n}}\left[\left\langle J_{G}|\mathbf{J}| J_{p}\right\rangle\right],
$$

where we denoted the position-dependent energy

$$
\epsilon_{p}^{n}=-\beta \Phi_{G}^{*}\left(\mathbf{R}_{n}\right) \Phi_{p}\left(\mathbf{R}_{n}\right) \sim-\beta / V .
$$

Consider first that the magnetic field is small such that the total effective field in Eq. (20) is dominated by the Knight field. The local impurity quantization axis is then collinear with the local particle spin direction $R_{\mathcal{B}^{n}}=R_{U_{n}}$, and Eqs. (43) and (45) give

$$
\begin{aligned}
H_{\mathrm{eff}}= & \sum_{n} \mathcal{B}^{n} \tilde{\mathbf{I}}_{z}^{n}+\sum_{p \in \uparrow} \sum_{n, m} \frac{\epsilon_{G}^{n} \epsilon_{p}^{m}}{E_{G}-E_{p}} \tilde{\mathbf{I}}_{z}^{n} \tilde{\mathbf{I}}_{z}^{m} \\
& +\sum_{p \in \downarrow} \sum_{n, m} \frac{\epsilon_{G}^{n} \epsilon_{p}^{m}}{E_{G}-E_{p}}\left(\tilde{\mathbf{I}}_{-}^{n} \tilde{\mathbf{I}}_{+}^{m}+\tilde{\mathbf{I}}_{-}^{m} \tilde{\mathbf{I}}_{+}^{n}\right) / 2 .
\end{aligned}
$$

We have split the summation over the particle excited states into those with the same and the opposite spin as is the spin of the ground state, corresponding to the second and the third terms in Eq. (47), respectively. Equation (47) makes it clear that there is a conserved quantity even in the presence of spin-orbit coupling, although it is neither the energy nor the total spin along any axis; it is the number of impurity spins locally aligned with the particle spin, equal to $\sum_{n} \tilde{I}_{z}^{n}$. This result is very general, as it is based only on the form of the spin-orbit coupling, which gives a single unitary operator $U$ for the whole particle spectrum. Restricting to the lowest excited state, as in Eq. (44), we get the standard result ${ }^{73,74}$

$$
H_{\mathrm{eff}}=\sum_{n} \mathcal{B}^{n} \tilde{I}_{z}^{n}-\sum_{n, m} \frac{\epsilon_{G}^{n} \epsilon_{G}^{m}}{E_{z}}\left(\tilde{\mathbf{I}}_{-}^{n} \tilde{\mathbf{I}}_{+}^{m}+\tilde{\mathbf{I}}_{-}^{m} \tilde{\mathbf{I}}_{+}^{n}\right) / 2,
$$

generalized to include the spin-orbit coupling effects. Without spin-orbit coupling, this result was first obtained in Ref. 73 and provided an important insight into the impurity-induced decoherence and its removal by spin echo, discussed below Eq. (44).

For the electronic case, we are, however, more interested in a different regime, where a finite magnetic field breaks the above-discussed symmetry and sets a global quantization axis for impurities, so that the Zeeman energy dominates in the total effective field in Eq. (20). We then have $R_{\mathcal{B}^{n}} \approx \mathbb{1}$ and $\tilde{\mathbf{I}} \approx \mathbf{I}$. Equation (45) can be then evaluated explicitly, using Eq. (7). Instead, we estimate the effects of weak spin-orbit coupling, which guarantees that $R_{m} \ll l_{\mathrm{SO}}$, by expanding the rotation operator up to the first order as

$$
\begin{gathered}
U\left(\mathbf{R}_{m}\right) \approx \mathbb{1}+O\left(r_{m} / l_{\mathrm{SO}}\right), \\
\tilde{I}_{+}^{n} \tilde{I}_{-}^{m}+\gamma \tilde{I}_{+}^{n} \tilde{I}_{+}^{m}+\gamma^{\prime} \tilde{I}_{+}^{n} \tilde{I}_{z}^{m}+\cdots,
\end{gathered}
$$

where $\gamma, \gamma^{\prime}=O\left[l / l_{\mathrm{SO}}\right]$. This is the most important message for the electron case, that the spin-orbit coupling results in the spin-nonconserving interactions in the impurity ensemble, which are, compared to the spin-conserving ones, suppressed by a position-dependent factor of the order of the ratio of the confinement and spin-orbit lengths.

We now turn attention to a hole dot, taking the lowest state in the heavy-hole subband as the ground state $G=\frac{3}{2}, 00,0$. The closest excited state, which gave by far the dominant contribution in the electronic case, is the spin opposite heavyhole state $p=-\frac{3}{2}, 00,0$. The corresponding vectors A scale as (see Appendix D for full expressions)

$$
\tilde{A}_{+}^{n} \sim \epsilon_{p}^{n} O\left(\lambda_{0}^{2}\right), \quad \tilde{A}_{-}^{n} \sim \epsilon_{p}^{n} \lambda_{0}, \quad \tilde{A}_{z}^{n} \sim \epsilon_{p}^{n} \lambda_{0} .
$$

To quantify the prefactor in the second-order term $\tilde{A}_{+}^{n}$, we would have to go to the next order in the perturbation expansion 
of the wave functions. However, this is not necessary as this term does not enter anywhere in the subsequent discussion. We conclude from Eq. (51) that the spin-conserving interactions mediated by the lowest heavy-hole excited state are proportional to the second power of parameters $\lambda$ (through terms such as $\left.\tilde{A}_{-} \tilde{A}_{-}^{*} \tilde{I}_{+} \tilde{I}_{-}\right)$, the same as the spin-nonconserving ones (e.g., $\tilde{A}_{-} \tilde{A}_{z}^{*} \tilde{I}_{+} \tilde{I}_{z}$ ). This is a drastic difference to the electron case, where the spin-conserving interactions dominate.

Let us now consider the light-hole subband. Taking $p=\frac{1}{2}$, 00,0, we get (see Appendix D)

$$
\tilde{A}_{+}^{n} \sim \epsilon_{p}^{n}, \quad \tilde{A}_{-}^{n} \sim \epsilon_{p}^{n} \lambda_{0}, \quad \tilde{A}_{z}^{n} \sim \epsilon_{p}^{n} \lambda_{1}^{\prime} .
$$

The light-hole excited state does mediate spin-conserving impurity interactions (through $\tilde{A}_{+} \tilde{A}_{+}^{*} \tilde{I}_{-} \tilde{I}_{+}$). Compared to these, the leading spin-nonconserving term $\left(\tilde{A}_{+} \tilde{A}_{z}^{*} \tilde{I}_{-} \tilde{I}_{z}\right)$ is suppressed linearly in $\lambda$. The energy denominator in the effective Hamiltonian is of the order of $100 \mathrm{meV}$ for the light-hole states (typical light-heavy-hole band offset) versus a few meV offset of the lowest heavy-hole excited state. For our parameters, this energy penalty is almost exactly compensated by much larger matrix elements for the spin-nonconserving interactions and more than compensated for the spin-conserving ones. We conclude that the light-hole ground state is the most efficient mediator of the spin-conserving interactions in the impurity ensemble, and a rather efficient mediator of the spin-nonconserving ones. As a direct consequence, and unlike for electrons, the decoherence induced by the hole-mediated evolution of the impurity bath will not be removed by the hole spin echo. This follows from Eq. (44) choosing $G=\frac{3}{2}$ and $p=\frac{1}{2}$ on one hand and $G=-\frac{3}{2}=T\left(\frac{3}{2}\right)$ and $p=-\frac{1}{2}=T\left(\frac{1}{2}\right)$ on the other. Since $E_{3 / 2}-E_{1 / 2} \approx E_{-3 / 2}-E_{-1 / 2}$, we get that the impurity interactions are independent (within this single excited state approximation) on the hole spin state.

\section{PHONON-INDUCED SPIN RELAXATION OF THE IMPURITY BATH}

We now use the results of the previous section to calculate how fast the impurity ensemble spin relaxes. The first and the second terms of the effective Hamiltonian (43) induce flip of a single impurity and a pair of impurities, respectively. For the former, terms with in-plane components of $\tilde{\mathbf{I}}$, while for the latter terms such as the last two terms in Eq. (50), are required for spin-nonconserving transitions. As the initialand final-state energies differ, in general, we consider that the transition is assisted by phonons, which provide for the energy conservation.

We consider several possible mechanisms as to how phonons can couple to the impurity bath and make order of magnitude estimates for the resulting relaxation rates. We find that the most efficient relaxation is due to the piezoelectric field spatially shifting the particle, leading to a $\mu$ s relaxation time for Mn spins. It is known that phonons are ineffective in relaxing nuclear spins; ${ }^{77}$ still we evaluate the resulting rates also for electrons because as we treated electrons and holes on the same footing, the formulas derived below apply for both. We find a $10^{11} \mathrm{~s}$ relaxation time for nuclear spins.

\section{A. Particle-phonon interactions}

The phonon-impurity interaction Hamiltonian $H_{i}$ is in general a function of the local lattice deformation arising in the presence of acoustic phonons:

$$
\delta \mathbf{R}=i \sum_{\mathbf{Q} \lambda} \sqrt{\frac{\hbar}{2 V_{0} \rho \omega_{\mathbf{Q} \lambda}}} \mathbf{e}_{\mathbf{Q} \lambda} e^{i \mathbf{Q} \cdot \mathbf{R}}\left(a_{\mathbf{Q} \lambda}+a_{-\mathbf{Q} \lambda}^{\dagger}\right) .
$$

Here, the phonon wave vector is $\mathbf{Q}$, polarization is $\lambda$ (one longitudinal $\lambda=l$ and two transversal ones $\left.\lambda=t_{1,2}\right)$ with $\mathbf{e}_{\mathbf{Q} \lambda}$ a real unit vector $\left(\mathbf{e}_{\mathbf{Q} \lambda}=-\mathbf{e}_{-\mathbf{Q} \lambda}\right), V_{0}$ is the crystal volume, $\rho$ is the material density, $\hbar \omega_{\mathbf{Q} \lambda}=\hbar c_{\lambda} Q$ is the phonon energy, $c_{\lambda}$ is the phonon velocity, and $a_{\mathbf{Q} \lambda}^{\dagger}$ is the phonon creation operator.

In a polar material, such as GaAs, the lattice deformation is accompanied by a piezoelectric field, which is the gradient of the following potential:

$$
\begin{aligned}
V_{\mathrm{PZ}}= & -i \Xi \sum_{\mathbf{Q} \lambda} \sqrt{\frac{2 \hbar}{V_{0} \rho \omega_{\mathbf{Q} \lambda}}} \frac{1}{Q^{2}} e^{i \mathbf{Q} \cdot \mathbf{R}}\left(a_{\mathbf{Q} \lambda}+a_{-\mathbf{Q} \lambda}^{\dagger}\right) \\
& \times\left[Q_{x} Q_{y}\left(\mathbf{e}_{\mathbf{Q} \lambda}\right)_{z}+Q_{z} Q_{x}\left(\mathbf{e}_{\mathbf{Q} \lambda}\right)_{y}+Q_{y} Q_{z}\left(\mathbf{e}_{\mathbf{Q} \lambda}\right)_{x}\right],
\end{aligned}
$$

with $\Xi$ the piezoelectric constant.

The lattice deformation also shifts the electronic bands, which is quantified by the deformation potential $V_{\mathrm{DP}}=$ $-\sigma \operatorname{div} \delta \mathbf{R}$. Equation (53) gives

$$
V_{\mathrm{DP}}=\sigma \sum_{\mathbf{Q}} \sqrt{\frac{\hbar}{2 V_{0} \rho \omega_{\mathbf{Q} l}}} Q e^{i \mathbf{Q} \cdot \mathbf{R}}\left(a_{\mathbf{Q} l}+a_{-\mathbf{Q} l}^{\dagger}\right),
$$

with $\sigma$ the deformation potential constant.

As we will see, a relative shift of the impurity and the particle, which we denote by d, induces impurity-phonon coupling, leading to the impurity-spin relaxation. Since impurities are tied to atoms, the phonon displacement is obviously such a relative shift $\mathbf{d}=\delta \mathbf{R}$, which we call "geometric." However, the phonon-induced electric fields $\mathbf{E}$ also lead to shifts. Namely, adding the potential of an in-plane field to that in Eq. (2) amounts to a shift of the quantum dot position by $\mathbf{d}=e \mathbf{E} l^{2} / \hbar \omega$ (electrically induced shifts along the perpendicular direction are much smaller, as the wave function is much stiffer along $z$ due to a stronger confinement). If the particle follows these potential changes adiabatically, which we assume, such a shift is equivalent to the shift of the impurities, fixed to the lattice, by $-\mathbf{d}$. Since the phonon electric fields are proportional to the displacement $\delta \mathbf{R}$, we can write a general expression

$$
|\mathbf{d}| \sim \alpha|\delta \mathbf{R}|,
$$

with a dimensionless factor $\alpha$. For the geometric shift mechanism, $\alpha=1$ by definition. For the piezoelectric field, comparing Eqs. (53) and (54), we get

$$
\alpha=2 Q \Xi l^{2} / \hbar \omega \text {. }
$$

Finally, the deformation potential gives

$$
\alpha=\sigma(Q l)^{2} / \hbar \omega \text {. }
$$

We evaluate $\alpha$ in Table I. As it enters the relaxation rates in the second power (see below), we can immediately quantify the relative importance of the three considered channels. Piezoelectric field is the most effective, for both electron and 
TABLE I. Values for the dimensionless coefficient $\alpha$, the ratio of the induced impurity shift versus the phonon displacement for various shift mechanisms (columns) and particles (rows). Parameters from Appendix E were used ( $\mathrm{GaAs}$ and $\mathrm{ZnTe}$ for the electron and hole cases, respectively); the phonon wave vector for electronic case was specified choosing $B=1 \mathrm{~T}$.

\begin{tabular}{lccc}
\hline \hline$\alpha$ & Piezoelectric & Deformation & Geometric \\
\hline Electron & 46 & 0.0038 & 1 \\
Hole & 38 & 17 & 1 \\
\hline \hline
\end{tabular}

hole cases, inducing shifts almost two orders of magnitude larger than the geometric shift. The electric field from the deformation potential is comparable to the piezoelectric for holes, and much smaller for electrons, which are deeply in the long phonon wavelength limit $Q l \ll 1$. We note that the geometric shifts will be in fact somewhat more effective than it seems from the table, as they may (unlike the electric fields) shift the wave function along the perpendicular direction. Such shifts can be described with an effective enhancement of $\alpha$ by a factor of $\pi l / w$, which, however, is not large enough to change the order of importance following from Table I.

We calculate the relaxation rate $\Gamma$ due to a general phononimpurity interaction $H_{i}$ by the Fermi's golden rule. For a given phonon polarization $\lambda$, it reads as

$$
\Gamma=\frac{2 \pi}{\hbar} \sum_{\mathbf{Q}}\left|\left\langle\mathcal{I}^{\prime}\left|H_{i}\right| \mathcal{I}\right\rangle\right|^{2} \delta\left(E_{\mathcal{I}}-E_{\mathcal{I}^{\prime}}-\hbar \omega_{\mathbf{Q} \lambda}\right) N_{Q},
$$

where $\mathcal{I}$ and $\mathcal{I}^{\prime}$ denote the initial and final states of the impurities, and we are interested in transitions where these two states differ in spin. The phonon occupation factor $N_{Q}=n_{Q}+1$, and $N_{Q}=n_{Q}$, if the energy of the initial state is larger, and smaller than the final state, respectively, with $n_{Q}=1 /\left[\exp \left(\hbar \omega_{\mathrm{Q} \lambda} / k_{B} T\right)-1\right]$. The energy conservation fixes the phonon wave-vector magnitude to $\left|E_{\mathcal{I}}-E_{\mathcal{I}^{\prime}}\right|=\hbar c_{\lambda} Q$, by which we get

$$
\Gamma=\frac{V_{0} Q^{2}}{\pi \hbar^{2} c_{\lambda}} N_{Q} \overline{\left|\left\langle\mathcal{I}^{\prime}\left|H_{i}\right| \mathcal{I}\right\rangle\right|^{2}} .
$$

The overline denotes the angular average

$$
\overline{f(\mathbf{Q})}=(1 / 4 \pi) \int d \Omega f(\mathbf{Q})
$$

over directions of the phonon wave vector $\mathbf{Q}$.

\section{B. Spin-phonon coupling due to impurity shift}

Assuming the shifts are small, we get the impurity-phonon coupling as

$$
H_{i}=-\left.\sum_{n} \mathbf{d} \cdot \frac{\partial H_{\mathrm{eff}}}{\partial \mathbf{R}}\right|_{\mathbf{R}=\mathbf{R}_{n}} .
$$

To calculate the spatial derivative of the effective Hamiltonian (43), it is easier to first evaluate the derivative of vectors $\mathcal{B}$ and $\mathbf{A}$ in the original coordinate system which does not depend on the position and then to transform them into the locally rotated coordinates.

Take the first term of the effective Hamiltonian (43). The finite derivative of the total field (20) is due to the spatial dependence of the Knight field

$$
\left(\mathbf{d} \cdot \partial_{\mathbf{R}}\right) \mathcal{B}^{n}=\left(\mathbf{d} \cdot \partial_{\mathbf{R}}\right) \mathbf{K}^{n},
$$

transversal components of which give the spin-increasing transition rate for impurity $n$ as

$$
\Gamma^{(1)}=\frac{V_{0} Q^{2}}{\pi \hbar^{2} c_{\lambda}} N_{Q} \overline{\left[\mathbf{r}_{-}^{\prime} \cdot\left(\mathbf{d} \cdot \partial_{\mathbf{R}}\right) \mathbf{K}\right]^{2}}\left|\left\langle I^{n+1}\left|\tilde{I}_{+}^{n}\right| I^{n}\right\rangle\right|^{2},
$$

and an analogous expression follows swapping the subscripts \pm for a spin-decreasing transition. To get an order of magnitude estimate for the rates as that in Eq. (64), we adopt the following simplifications: We replace the matrix elements of the raising/lowering operators by

$$
\left|\left\langle I^{n \pm 1}\left|\tilde{I}_{ \pm}^{n}\right| I^{n}\right\rangle\right|^{2}=I(I+1)-I^{n}\left(I^{n} \pm 1\right) \sim I^{2},
$$

choose the direction of the phonon wave vector that gives the highest contribution, instead of performing the angular average in Eq. (61), and denote ( $\delta$ equals $l$ and $w / \pi$ for an in-plane and out-of-plane polarized phonon, respectively, see Appendix C)

$$
\nabla \tilde{\mathbf{K}}_{-} \equiv \delta^{-1} \overline{\mathbf{r}_{-}^{\prime} \cdot\left(\mathbf{d} \cdot \partial_{\mathbf{R}}\right) \mathbf{K}} .
$$

Finally, we use Eq. (56) and $\delta R \sim \sqrt{ } \hbar / 2 V_{0} \rho c_{\lambda} Q$ to write

$$
\Gamma^{(1)} \sim \frac{\alpha^{2} I^{2} N_{Q} Q}{2 \pi \hbar c_{\lambda}^{2} \rho}\left(\nabla \tilde{\mathbf{K}}_{-}\right)^{2},
$$

a general form for the relaxation rate estimate, which we evaluate now for specific cases.

Let us start with the electronic case. The transition energy is dominated by the external field $\hbar c_{\lambda} Q \approx\left|g \mu_{N} B\right|$ and is much smaller than the thermal energy, so that $N_{Q} \approx k_{B} T / \hbar c_{\lambda} Q$. We evaluate the derivative of the Knight field in Appendix $C$ [see Eq. (C6)], getting

$$
\nabla \tilde{\mathbf{K}}_{ \pm} \sim(\beta / V) l_{\mathrm{SO}}^{-1} .
$$

For the dominant piezoelectric mechanism, we get

$$
\Gamma^{(1)} \sim \frac{9 I^{2}}{2 \pi^{3}} \frac{k_{B} T\left(g \mu_{N} B\right)^{2}}{\hbar E_{\mathrm{PZ}}^{2}} \frac{l^{4}}{w^{2} l_{\mathrm{SO}}^{2}},
$$

where the energy $E_{\mathrm{PZ}}=\sqrt{\hbar^{7} c_{\lambda}^{5} \rho} / \Xi m \beta$ is a material constant. Evaluating parameters of GaAs, we get a minuscule rate $\Gamma^{(1)} \sim$ $2 \times 10^{-11} \mathrm{~s}^{-1}$, choosing transversal phonons, external field $1 \mathrm{~T}$, and temperature $1 \mathrm{~K}$.

We now turn to holes. The transferred energy is now given by the Knight field $\left|E_{\mathcal{I}}-E_{\mathcal{I}^{\prime}}\right| \sim J \beta / V$ and we again consider a high-temperature limit $k_{B} T \geqslant\left|E_{\mathcal{I}}-E_{\mathcal{I}^{\prime}}\right|$. As we show in Appendix C, the result in Eq. (68) is changed into

$$
\nabla \tilde{\mathbf{K}}_{ \pm} \sim(\beta / V)\left(\sqrt{3} \lambda_{1} / l\right),
$$

showing that quantities of the form $l / \lambda$ can be seen as effective spin-orbit lengths for holes. Equation (69) can be then used putting for the "spin-orbit length" the one just described and replacing the external Zeeman energy by the Knight field. For convenience, we give the relaxation rate explicitly

$$
\Gamma^{(1)} \sim \frac{3^{5} I^{2} J^{2}}{2^{5} \pi^{4}} \frac{k_{B} T}{\hbar} \frac{\beta^{2}}{E_{\mathrm{PZ}}^{2}} \frac{\lambda_{1}^{2}}{w^{4} l^{2}},
$$

which for ZnTe parameters and temperature $10 \mathrm{~K}$ yields a modest rate $0.35 \mu \mathrm{s}^{-1}$. The rate is second order in the 
"spin-orbit strength" and, unlike for electrons, grows fast upon making the dot smaller since now the effect of Knight field, inversely proportional to the dot volume, dominates over the effect of the shift being larger for softer dot potential.

We now turn to the second part of the effective Hamiltonian (43). Phonon-induced fluctuations of this part enable a simultaneous change of spin of two impurities. Comparing the two terms of the effective Hamiltonian, and using the results of Appendix D, the pairwise spin transition mediated by the lowest heavy-hole excited state relates to the single spin-flip rate by

$$
N \Gamma_{p=3 / 2}^{(2)} / \Gamma^{(1)} \sim N I^{2}\left(2 \frac{\lambda_{0}^{2}}{\lambda_{1}} \frac{\beta / V}{E_{\downarrow}-E_{\uparrow}}\right)^{2},
$$

which evaluates to $10^{-2}$. Similarly, we get for the mediation through the light-hole state

$$
N \Gamma_{p=1 / 2}^{(2)} / \Gamma^{(1)} \sim N\left(\frac{3 \lambda_{1}^{\prime}}{4 \lambda_{1}} \frac{\beta / V}{\Delta_{\mathrm{lh}}}\right)^{2},
$$

where a much larger energy offset $\Delta_{\mathrm{lh}}$ is partially compensated by a larger matrix element. The spin relaxation rate of the impurity ensemble scales as $\Gamma^{(1)}$ and $N \Gamma^{(2)}$ for the single and pairwise flip channels, respectively, because the number of pairs available for a flip is of order $N^{2}$. This is why we inserted the factor $N$ in the previous two equations. This fact also suggests a possible experimental discrimination for the two channels through their dependence on the impurity density. The ensemble relaxation through single (pairwise) flips is insensitive (linearly proportional) to the impurity density.

For completeness, we note that for electrons a similar relation between the first- and second-order rates holds,

$$
\Gamma^{(2)} \sim \Gamma^{(1)}\left(\frac{\beta / V}{E_{\downarrow}-E_{\uparrow}}\right)^{2} I^{2} \ll \Gamma^{(1)},
$$

but the ratio is much smaller, at the external field of $1 \mathrm{~T}$ by 10 orders of magnitude.

We now consider additional mechanisms of the phononimpurity couplings, through which impurity-spin relaxation may arise.

\section{Valence band shifts}

The phonon-induced lattice compression changes the band structure: the bands are shifted. Shifts different for the bands of the particle ground and mediating excited states $p$ result in the impurity-phonon coupling through the second term of Eq. (43) by changing the denominator by $\Delta V_{\mathrm{DP}}$. As the two states have to belong to different bands, such a coupling may arise only for the case of holes and takes the form

$$
H_{i} \sim \frac{\Delta V_{\mathrm{DP}}}{\Delta_{\mathrm{lh}}} H_{\mathrm{eff}},
$$

expanding the effective Hamiltonian up to the lowest order in the band-shift difference $\Delta V_{\mathrm{DP}}=-\left(\sigma_{\mathrm{hh}}-\sigma_{\mathrm{lh}}\right) \operatorname{div} \delta \mathbf{R}$, which is the difference of the deformation potentials for the heavyand light-hole valence bands. We can thus relate the bandshift mechanism to the position-shift one, comparing Eq. (62) with (75). We find that the latter is described by an effective constant $\alpha$ :

$$
\alpha_{\mathrm{eff}}=\left(\sigma_{\mathrm{hh}}-\sigma_{\mathrm{lh}}\right) \sigma Q l / \Delta_{\mathrm{lh}} .
$$

If we estimate the potential difference by the typical value of the potential itself $\left(\sigma_{\mathrm{hh}}-\sigma_{\mathrm{lh}}\right) \sim 5 \mathrm{eV}$, which is likely an overestimated value, we get $\alpha_{\text {eff }}=17$, so that the coupling through band shifts leads to a rate at most comparable to (and most probably much smaller than) that described by Eq. (73).

\section{Renormalization of the spin-orbit length}

Phonon-induced renormalization of band offsets influences the spin-orbit couplings. This is evident from the expressions for the coefficients $\lambda$ which are inversely proportional to the light-heavy-hole offset $\Delta_{\text {lh }}$ [see Eqs. (A9)]. Even though the coupling in the form of the constant $\alpha$ is described by the same formula as in Eq. (76), the substantial difference is that now the phonon-impurity coupling arises also through the first term of the effective Hamiltonian because fluctuations in spin-orbit fields induce fluctuations in the Knight field. The corresponding $\alpha_{\text {eff }}$ is the one given in Eq. (76) and the relaxation rate is that in Eq. (67), so that it does not exceed the rate due to the piezoelectric shift mechanism. For the case of electrons, the effective constant follows in an analogous form

$$
\alpha=\left(\sigma_{e}-\sigma_{h}\right) \sigma Q l_{\mathrm{SO}} / \Delta,
$$

where $\Delta$ is of the order of the conduction to valence band offset, which enters the definition of the spin-orbit couplings $1 / l_{\text {SO }}$. The numerical value for $\alpha$ is much less than one even taking $\left(\sigma_{e}-\sigma_{h}\right) \sim \sigma_{e}$, so that this channel is negligible with respect to the geometrical shifts.

\section{E. Phonon-induced spin-orbit interactions}

Finally, we estimate the influence of spin-phonon coupling through an additional spin-orbit interaction arising in the presence of a phonon-originated electric field. We assume the new spin-orbit interaction strength relates to the one we considered in Sec. II, referred to as "old," through the ratio of the internal (interface) electric field $E_{\text {int }}$ and the phonon-induced electric field, the latter given as the gradient of the appropriate potential [Eqs. (54) or (55)]. Since the phonon-induced spin-orbit interaction ("new") arises from an electric field, it is of the Rashba functional form, and one can take its effects to be additive to the "old" one. For electrons, this means $U_{\text {tot }}=U_{\text {old }} U_{\text {new }} \approx\left(\mathbb{1}+O_{\text {old }}\right)\left(\mathbb{1}+O_{\text {new }}\right) \approx$ $\mathbb{1}+\left(O_{\text {old }}+O_{\text {new }}\right)$, which amounts to additive inverse of the spin-orbit lengths $1 / l_{\mathrm{SO}} \rightarrow 1 / l_{\mathrm{SO}}+1 / l_{p h \text {-SO }}$. By inspecting Eq. (C5), we estimate the effective interaction to be described again by Eq. (67) with the constant

$$
\alpha_{\mathrm{eff}}=\Xi Q l / e E_{\mathrm{int}}
$$

for the piezoelectric phonon field (a much smaller deformation field corresponds to the numerator replaced by $\sigma Q^{2} l$ ). The numerator evaluates to $10^{6} \mathrm{~V} / \mathrm{m}$, which is not supposed to be much higher than the internal field, so that again we find that this mechanism is less important than the one due to the piezoelectric shift. We come to a similar conclusion for 
holes. Namely, even though the phonon-induced piezoelectric field is much stronger, of the order of $10^{8} \mathrm{~V} / \mathrm{m}$, the spin-orbit terms in the Kohn-Luttinger Hamiltonian are less effective in inducing light-heavy-hole mixing than the terms we considered explicitly in previous sections (see Appendix F for details).

\section{CONCLUSIONS}

We have analyzed the interactions within an ensemble of impurity spins, which are mediated by a confined spin-orbit coupled particle. We have considered two physical systems where such mediated interactions are of great importance: III-V (GaAs) electronic lateral quantum dot with nuclear spins as impurities, and II-VI (ZnTe) self-assembled Mn-doped quantum dot populated by a hole. Our focus has been on the consequences of the spin-orbit coupling on the character of the mediated interactions.

We have derived an effective Hamiltonian for the impurity ensemble, treating the particle-impurity interaction perturbatively. The form of this Hamiltonian allowed us to quantify the degree to which the conservation of impurities spin is broken in the presence of the spin-orbit coupling of the particle. We have found that for the electron case, the spin-nonconserving terms are suppressed relative to the spin-conserving ones by a small factor, the ratio of the confinement length, and the spin-orbit length. The lowest electron excited state is the most effective mediator, which results in a decoherence being removable by the electron spin echo even in the presence of the spin-orbit coupling. In the case of holes, the spin-conserving interactions are most efficiently mediated by the lowest light-hole state, while the spin-nonconserving ones by the lowest heavy-hole state. The induced decoherence is then not removable by a hole spin echo anymore.

As a direct application of the derived effective Hamiltonian, we have calculated the rates of a phonon-assisted impurity spin relaxation, which arises only in the presence of the spin-orbit coupling in the particle Hamiltonian. We have considered several coupling mechanisms by which impurity spins couple to phonons. We have found that the most effective is the piezoelectric field induced shift of the particle wave function, with a typical relaxation time of $1 \mu \mathrm{s}$ in a $10-\mathrm{nm}$ self-assembled strain-free quantum dot. The rate grows upon making the dot smaller, or diminishing the heavy-light-hole splitting by strain, possibly to nanoseconds for reasonable dot parameters. Furthermore, even when the identified $\mu \mathrm{s}$ time scale of spin-lattice relaxation falls short of explaining the observed fast magnetic polaron formation, it is still an important quantity that determines the time scale on which the magnetization can be switched. Namely, the polaron formation is a complex process involving several stages with different time scales and mechanisms. ${ }^{78}$ The rates we calculate (the spin-lattice relaxation times) are those taking place at the end (and after) the polaron formation. Although the final answer has not been given yet, the latter is, most probably, due to the direct (or super) exchange between Mn spins assisted by atomic spin-orbit interactions in Mn clusters (see Ref. 79). This is to be contrasted with the mechanism we consider, where the spin dissipation is allowed by the valence band spin-orbit coupling of the confined particle.
While we have focused on a single particle, our analysis of the spin-nonconserving mechanisms already provides insights in the magnetic ordering in quantum dots with multiple occupancy. Our results are directly applicable for a dot occupied by a few noninteracting particles, where the impurity interaction is a sum of single-particle contributions. Interesting and qualitatively new effects are expected once interactions between the mediating particles are important, spanning from Kondo physics and nonperturbative renormalization of the Ruderman-Kittel-Kasuya-Yosida (RKKY) interaction ${ }^{80}$ to thermally enhanced magnetism ${ }^{81}$ and the nanoscale analog of the Wigner crystallization. ${ }^{68}$ In the transport studies, even the well-established concept of spin filtering with magnetic semiconductors ${ }^{82-85}$ can have important ramifications for nanoscale structures such as quantum dots. ${ }^{86}$ The spinnonconserving mechanisms are also the key in understanding the prediction of piezomagnetic quantum dots or, equivalently, nonlinear magneto-electric effects. ${ }^{87}$ Changes in the shape of lateral confinement (from circular to elliptical) controlled by the pair of the gate electrodes have been demonstrated experimentally to alter the particle configuration from vanishing to finite spin in nonmagnetic quantum dots. ${ }^{88}$ This principle provides possibilities for the control of magnetic ordering in dots with added Mn impurities where such changes in the particle spin, through exchange interaction, would reversibly control the magnetic ordering of the nearby $\mathrm{Mn}$ spins. ${ }^{87}$ The characteristic time scale for the related magnetic polaron formation, similar to the better studied quantum well structures, should be largely determined by the anisotropic spin-spin interactions of impurities which explicitly do not conserve the total spin of Mn atoms. ${ }^{43,56,89,90}$

Beyond epitaxial dots that we have presently examined, recent experimental advances in colloidal quantum dots warrant also future considerations. Typically, they are easily synthesized II-VI materials, such as ZnTe, ZnSe, CdS, and CdSe, ${ }^{91,92}$ which offer a large size-induced tunability of the transition energies and long spin decoherence times. ${ }^{93,94}$ Magnetic doping ${ }^{95}$ of these colloidal dots provides an opportunity for a versatile control of magnetic order as well as leads to robust magnetic polaron formation with effective internal magnetic field approaching $100 \mathrm{~T}^{43,95-99}$

\section{ACKNOWLEDGMENTS}

We would like to thank R. Oswaldowski for many discussions, which substantially contributed to this work. P.S. would also like to acknowledge useful discussions with $\mathrm{U}$. Zuelicke. This work was supported by EU project Q-essence, meta-QUTE ITMS NFP 26240120022, CE SAS QUTE, COQI APVV-0646-10, SCIEX, DOE-BES, ONR, and DFG SFB 689.

\section{APPENDIX A: KOHN-LUTTINGER HAMILTONIAN PERTURBATIVE EIGENSTATES}

Here, we derive hole eigenstates in the lowest-order perturbation theory. We neglect the influence of the conduction and spin-orbit split-off subbands and consider only the light $\left(J= \pm \frac{1}{2}\right)$ and heavy $\left(J= \pm \frac{3}{2}\right)$ holes in the Kohn-Luttinger 
Hamiltonian ${ }^{100} H_{J J^{\prime}}$. The diagonal elements are

$$
\begin{aligned}
& H_{ \pm 3 / 2, \pm 3 / 2}=-\frac{\hbar^{2}}{2 m_{0}}\left[k_{z}^{2}\left(\gamma_{1}-2 \gamma_{2}\right)+\left(k_{x}^{2}+k_{y}^{2}\right)\left(\gamma_{1}+\gamma_{2}\right)\right] \\
& H_{ \pm 1 / 2, \pm 1 / 2}=-\frac{\hbar^{2}}{2 m_{0}}\left[k_{z}^{2}\left(\gamma_{1}+2 \gamma_{2}\right)+\left(k_{x}^{2}+k_{y}^{2}\right)\left(\gamma_{1}-\gamma_{2}\right)\right]
\end{aligned}
$$

with $\hbar \mathbf{k}=\mathbf{P}$ the hole momentum operator, $m_{0}$ the free-electron mass, and $\gamma_{1}, \gamma_{2}$, and $\gamma_{3}$ (below) the Luttinger parameters. Together with the in-plane $V(\mathbf{r})$ and heterostructure $V_{z}(z)$ confinement potentials, assumed to be those in Eq. (2), the kinetic terms in Eq. (A1) define the dot unperturbed eigenstates (normalization omitted)

$$
\Phi_{n m, k}^{J}=r^{|m|} e^{-r^{2} / 2 l_{J}^{2}} L_{n}^{|m|}\left(r^{2} / l_{J}^{2}\right) e^{i m \phi} \sin (k \pi z / w) .
$$

We used cylindrical coordinates $(r, \phi, z), L_{n}^{m}$ are the associated Laguerre polynomials, $l_{J}$ the in-plane confinement length (which differs for heavy and light holes due to their different masses), and $w$ the heterostructure width. The Fock-Darwin states are labeled by the principal and orbital quantum numbers $n$ and $m$, and $k$ labels excitations in the perpendicular potential. The corresponding energies are

$$
E_{J, n m, k}=\hbar \Omega_{J}(2 n+|m|+1)+\frac{\hbar^{2}}{2 m_{J} w^{2}} \pi^{2} k^{2},
$$

where the in-plane excitation energy is parametrized by the mass and confinement length

$$
\hbar \Omega_{J}=\frac{\hbar^{2}}{m_{J} l_{J}^{2}} .
$$

The in-plane masses are given by Eq. (A1): $m_{ \pm 3 / 2} \equiv m_{\mathrm{hh}}=$ $m_{0} /\left(\gamma_{1}+\gamma_{2}\right), m_{ \pm 1 / 2} \equiv m_{\mathrm{lh}}=m_{0} /\left(\gamma_{1}-\gamma_{2}\right)$. We parametrize the in-plane electrostatic potential choosing a value for the heavy-hole in-plane excitation energy $E_{3 / 2,01,0}-E_{3 / 2,00,0}=$ $\hbar \Omega_{\mathrm{hh}}$, which then specifies the confinement lengths. The lighthole excitation energy and confinement length

$$
\Omega_{\mathrm{lh}}=\Omega_{\mathrm{hh}}\left(m_{\mathrm{hh}} / m_{\mathrm{lh}}\right)^{1 / 2}, \quad l_{\mathrm{lh}}=l_{\mathrm{hh}}\left(m_{\mathrm{hh}} / m_{\mathrm{lh}}\right)^{1 / 4}
$$

differ from the corresponding heavy-hole quantities due to a different in-plane mass. The energies of the hard-wall eigenstates also differ for heavy and light holes due to different out-of-plane masses, which are $m_{0} /\left(\gamma_{1}+2 \gamma_{2}\right)$ and $m_{0} /\left(\gamma_{1}-2 \gamma_{2}\right)$, respectively. We set $w$ by choosing a value for the light-heavy-hole offset $E_{3 / 2,00,0}-E_{1 / 2,00,0}=\Delta_{\mathrm{lh}}$.

Taking the above eigenstates as the basis, we now perturbatively take into account the off-diagonal elements of the Hamiltonian

$$
\begin{aligned}
& H_{ \pm 3 / 2, \pm 1 / 2}= \pm \frac{\hbar^{2}}{2 m_{0}} 2 \sqrt{3} \gamma_{3} k_{\mp} k_{z}, \\
& H_{ \pm 3 / 2, \mp 1 / 2}=\frac{\hbar^{2}}{2 m_{0}} \sqrt{3}\left[\gamma_{2}\left(k_{x}^{2}-k_{y}^{2}\right) \mp 2 \mathrm{i} \gamma_{3} k_{x} k_{y}\right], \\
& H_{ \pm 3 / 2, \mp 3 / 2}=0=H_{ \pm 1 / 2, \mp 1 / 2 .}
\end{aligned}
$$

We employ the nondegenerate perturbation theory

$$
\left|\Psi_{J a}\right\rangle=|J\rangle \otimes\left|\Phi_{a}^{J}\right\rangle+\sum_{J^{\prime} a^{\prime} \neq J a} \frac{\left\langle\Phi_{a^{\prime}}^{J^{\prime}}\left|H_{J^{\prime} J}\right| \Phi_{a}^{J}\right\rangle}{E_{J a}-E_{J^{\prime} a^{\prime}}}\left|J^{\prime}\right\rangle \otimes\left|\Phi_{a^{\prime}}^{J^{\prime}}\right\rangle,
$$

where we use the notation introduced below Eq. (4), so that $a$ includes two quantum numbers of the in-plane Fock-Darwin state and one of the perpendicular hard-wall state. To proceed, we neglect high-energy excitations $n>0$ and $k>1$ and adopt the axial approximation

$$
H_{ \pm 3 / 2, \mp 1 / 2} \approx \frac{\hbar^{2}}{2 m_{0}} \sqrt{3} \gamma k_{\mp}^{2},
$$

with $\gamma=\left(\gamma_{2}+\gamma_{3}\right) / 2$. With these simplifications, the otherwise infinite sum for $\left|\Psi_{J a}\right\rangle$ simplifies to only a single term for each $J^{\prime} \neq J$ and can be given explicitly. ${ }^{101,102}$ We finally get Eq. (9) with the admixtures

$$
\begin{aligned}
& \lambda_{1}=\frac{\hbar^{2}}{m_{0} l_{\mathrm{lh}} w} \frac{\gamma_{3} \sqrt{3} \kappa \xi}{\Delta^{*}+\hbar \Omega_{\mathrm{lh}}}, \\
& \lambda_{0}=\frac{\hbar^{2}}{m_{0} l_{\mathrm{lh}}^{2}} \frac{\gamma \sqrt{3 / 2} \kappa}{\Delta_{\mathrm{lh}}+2 \hbar \Omega_{\mathrm{lh}}},
\end{aligned}
$$

where $\Delta^{*}=E_{1 / 2,00,1}-E_{3 / 2,00,0}$ is the $z$-excited light-hole offset and

$$
\kappa=\left\langle\Phi_{00,0}^{\mathrm{hh}} \mid \Phi_{00,0}^{\mathrm{lh}}\right\rangle=\frac{2}{\left(m_{\mathrm{hh}} / m_{\mathrm{lh}}\right)^{1 / 4}+\left(m_{\mathrm{lh}} / m_{\mathrm{hh}}\right)^{1 / 4}}
$$

is the ground-state overlap. Finally, the dimensionless matrix element $\xi$ is defined by $\xi=-i w\left\langle 1\left|k_{z}\right| 0\right\rangle=\frac{8}{3}$.

The admixture of a $\frac{1}{2}$ light hole into a $\frac{3}{2}$ heavy hole scales as $1 / w l$, costs the in-plane plus perpendicular orbital energy (the latter is larger than the light-heavy-hole offset), and leads to an admixture with a very different $z$ profile than the main wave-function component. The admixture of the $-\frac{1}{2}$ light hole has a smaller numerator, proportional to $1 / l^{2}$, but costs only the in-plane orbital energy (several times smaller than the lightheavy-hole offset) and has the same $z$ profile as the main component.

Along the same lines, we get Eq. (11) with

$$
\begin{aligned}
& \lambda_{1}^{\prime}=\frac{\hbar^{2}}{m_{0} l_{\mathrm{hh}} w} \frac{\gamma_{3} \sqrt{3} \kappa \xi}{\Delta^{* \prime}+\hbar \Omega_{\mathrm{hh}}}, \\
& \lambda_{0}^{\prime}=\frac{\hbar^{2}}{m_{0} l_{\mathrm{hh}}^{2}} \frac{\gamma \sqrt{3 / 2} \kappa}{\Delta_{\mathrm{lh}}-2 \hbar \Omega_{\mathrm{hh}}},
\end{aligned}
$$

where $\Delta^{* \prime}=E_{3 / 2,00,1}-E_{1 / 2,00,0}$ is the z-excited heavy-hole offset.

For completeness, we list the hole Zeeman term ${ }^{103}$

$$
H_{h Z}=2 \kappa \mu_{B} \mathbf{J} \cdot \mathbf{B}+2 q \mu_{B} \sum_{i=x, y, z} J_{i}^{3} B_{i},
$$

which can be written for the heavy-hole subspace as

$$
H_{\mathrm{hh}, Z}=g_{\mathrm{hh}} \mu_{B}[\mathbf{J} / 3]_{z} \cdot \mathbf{B}_{z},
$$

with $\mathbf{J} / 3 \equiv \sigma / 2$ the pseudospin operator and $g_{\text {hh }} \equiv 6 \kappa+$ $27 q / 2 \approx 2$ for GaAs. ${ }^{102}$

\section{APPENDIX B: SPIN MATRIX ELEMENTS FOR HOLES}

Here, we calculate the matrix elements of the spin operator between hole perturbative eigenstates. For the purposes of this Appendix, we shorten the expression in Eq. (9), introducing 


$$
\begin{aligned}
\Phi=\Phi_{00,0}^{\mathrm{hh}}, a & =\lambda_{1} \Phi_{01,1}^{\mathrm{lh}}, \text { and } b=\lambda_{0} \Phi_{02,0}^{\mathrm{lh}} \text { to } \\
\left|\Psi_{3 / 2}\right\rangle & =\Phi|3 / 2\rangle+a|1 / 2\rangle+b|-1 / 2\rangle, \\
\left|\Psi_{-3 / 2}\right\rangle & =\Phi^{*}|-3 / 2\rangle-a^{*}|-1 / 2\rangle+b^{*}|1 / 2\rangle .
\end{aligned}
$$

We denote $J_{ \pm}=J_{x} \pm \mathrm{i} J_{y}$, so that $J_{x}=\left(J_{+}+J_{-}\right) / 2$ and $J_{y}=\left(J_{+}-J_{-}\right) / 2 \mathrm{i}$. Since the orbital operator in all the matrix elements is the delta function $\delta\left(\mathbf{R}-\mathbf{R}_{n}\right)$, all the complex amplitudes below should be evaluated at the position of the particular impurity, e.g., $\Phi \rightarrow \Phi\left(\mathbf{R}_{n}\right)$. Listing only the leading order in small quantities $\lambda_{0,1}$, to which $a$ and $b$ are proportional, we have

$$
\begin{aligned}
\left\langle\Psi_{3 / 2}\left|J_{z}\right| \Psi_{3 / 2}\right\rangle & =(3 / 2)|\Phi|^{2}+O\left(\lambda^{2}\right), \\
\left\langle\Psi_{3 / 2}\left|J_{+}\right| \Psi_{3 / 2}\right\rangle & =\sqrt{3} \Phi^{*} a+O\left(\lambda^{2}\right), \\
\left\langle\Psi_{3 / 2}\left|J_{-}\right| \Psi_{3 / 2}\right\rangle & =\sqrt{3} \Phi a^{*}+O\left(\lambda^{2}\right),
\end{aligned}
$$

from where Eq. (19) follows directly. The time-reversal symmetry gives (using $\langle T a \mid b\rangle=-\langle a \mid T b\rangle^{*}$, and $T^{2}=-1$ )

$$
\left\langle\Psi_{-3 / 2}|\mathbf{J}| \Psi_{-3 / 2}\right\rangle=-\left\langle\Psi_{3 / 2}|\mathbf{J}| \Psi_{3 / 2}\right\rangle,
$$

so that the spin expectation value changes sign upon inverting the hole spin. To evaluate the off-diagonal element of the Overhauser field and the vectors $\mathbf{A}$ we need

$$
\begin{aligned}
\left\langle\Psi_{-3 / 2}\left|J_{z}\right| \Psi_{3 / 2}\right\rangle & =a b, \\
\left\langle\Psi_{-3 / 2}\left|J_{+}\right| \Psi_{3 / 2}\right\rangle & =2 b^{2}, \\
\left\langle\Psi_{-3 / 2}\left|J_{-}\right| \Psi_{3 / 2}\right\rangle & =2 \sqrt{3} \Phi b+O\left(\lambda^{2}\right),
\end{aligned}
$$

from where Eq. (30) follows.

We use analogous shorthand notation for the light hole $\Phi^{\prime}=\Phi_{00,0}^{\mathrm{lh}}, a^{\prime}=\lambda_{1}^{\prime} \Phi_{0 \overline{1}, 1}^{\mathrm{hh}}$, and $b^{\prime}=\lambda_{0}^{\prime} \Phi_{0 \overline{2}, 0}^{\mathrm{hh}}$ to write

$$
\begin{aligned}
\left|\Psi_{1 / 2}\right\rangle & =\Phi^{\prime}|1 / 2\rangle+a^{\prime}|3 / 2\rangle-b^{\prime}|-3 / 2\rangle, \\
\left|\Psi_{-1 / 2}\right\rangle & =\Phi^{\prime *}|-1 / 2\rangle-a^{\prime *}|-3 / 2\rangle-b^{\prime *}|3 / 2\rangle .
\end{aligned}
$$

The light-hole-mediated effective interaction is given by

$$
\begin{aligned}
\left\langle\Psi_{3 / 2}\left|J_{z}\right| \Psi_{1 / 2}\right\rangle & =(3 / 2) \Phi^{*} a^{\prime}+(1 / 2) a^{*} \Phi^{\prime}, \\
\left\langle\Psi_{3 / 2}\left|J_{+}\right| \Psi_{1 / 2}\right\rangle & =\sqrt{3} \Phi^{*} \Phi^{\prime}+O\left(\lambda^{2}\right), \\
\left\langle\Psi_{3 / 2}\left|J_{-}\right| \Psi_{1 / 2}\right\rangle & =2 \Phi^{\prime} b^{*}+O\left(\lambda^{2}\right) .
\end{aligned}
$$

Finally, the coupling through the spin-opposite light-hole state is given by

$$
\begin{aligned}
\left\langle\Psi_{3 / 2}\left|J_{z}\right| \Psi_{-1 / 2}\right\rangle & =-(3 / 2) \Phi^{*} b^{\prime *}-(1 / 2) b^{*} \Phi^{* \prime} \\
\left\langle\Psi_{3 / 2}\left|J_{+}\right| \Psi_{-1 / 2}\right\rangle & =2 a^{*} \Phi^{*}+O\left(\lambda^{2}\right) \\
\left\langle\Psi_{3 / 2}\left|J_{-}\right| \Psi_{-1 / 2}\right\rangle & =-\sqrt{3} b^{\prime *} a^{*}
\end{aligned}
$$

\section{APPENDIX C: KNIGHT FIELD AND ROTATED COORDINATES}

Here, we evaluate the Knight field, its spatial derivative, and the corresponding locally rotated coordinate frame.

\section{Electron case}

The Knight field of an electron in the ground state is

$$
\mathbf{K}=-(\beta / 2)\left|\Phi_{G}(\mathbf{R})\right|^{2}\langle\mathbf{s}(\mathbf{R})\rangle
$$

[see Eq. (18)]. In deriving that, we used the identity

$$
U \mathbf{J} U^{\dagger}=R_{U}^{-1}[\mathbf{J}],
$$

where the unitary operator of spinor rotation

$$
U \equiv \exp (-i \mathbf{n} \cdot \mathbf{J} \phi)
$$

corresponds to a three-dimensional rotation around the unit vector $\mathbf{n}$ by angle $\phi$,

$$
R_{U}=\exp (-i \mathbf{n} \cdot \mathbf{l} \phi),
$$

with $\left(l_{k}\right)_{m n}=-i \epsilon_{k m n}$.

The spatial derivative of the Knight field, which gives the matrix elements for the impurity spin flips in the "first-order" rates, follows from Eq. (C1) as

$$
\nabla \mathbf{K}=\left[\nabla \ln \left(\left|\Phi_{G}(\mathbf{R})\right|^{2}\right)\right] \mathbf{K}+\left(\nabla \mathbf{n}_{\mathrm{so}}\right) \times \mathbf{K} .
$$

The two terms correspond, respectively, to the position change in the magnitude and direction of vector $\mathbf{K}$.

For electrons we consider a regime in which the total field is dominated by the external field. The impurity local coordinate system then coincides with the coordinate frame defined by the external magnetic field $R_{\mathcal{B}^{n}} \approx \mathbb{1}$ and $\hat{\mathbf{z}}^{\prime}=\mathbf{s}_{0}$. This allows us to estimate the components of $\nabla \mathbf{K}$ transversal to the local coordinate system as

$$
\mathbf{r}_{ \pm}^{\prime} \cdot \nabla \mathbf{K} \sim \delta^{-1}\left(l / l_{\mathrm{SO}}\right) K
$$

which originate in the first term of Eq. (C5) and where the length $\delta=l$ for a phonon with in-plane polarization vector $(\mathbf{e} \perp \hat{\mathbf{z}})$ and $\delta=w / \pi$ for a phonon with a polarization vector along the growth direction $(\mathbf{e} \| \hat{\mathbf{z}}$ ). The second term of Eq. (C5) gives a contribution at most as large as the first term, or lower, depending on the phonon polarization.

\section{Holes}

The Knight field of a spin- $\frac{3}{2}$ hole, defined by Eq. (17),

$$
\mathbf{K}^{n}=-\beta\left\langle\Psi_{3 / 2}\left|\delta\left(\mathbf{R}-\mathbf{R}_{n}\right) \mathbf{J}\right| \Psi_{3 / 2}\right\rangle,
$$

follows from Eqs. (B3) as

$$
\left[K_{x}^{n}, K_{y}^{n}, K_{z}^{n}\right]=-\beta\left[\sqrt{3} \operatorname{Re}\left(a \Phi^{*}\right), \sqrt{3} \operatorname{Im}\left(a \Phi^{*}\right), 3 / 2|\Phi|^{2}\right],
$$

given in the main text in Eq. (19), here using the notation from Appendix B $a=\lambda_{1} \Phi_{01,1}^{\mathrm{lh}}\left(\mathbf{R}_{n}\right)$ and $\Phi=\Phi_{00,0}^{\mathrm{hh}}\left(\mathbf{R}_{n}\right)$. Since for holes we are interested in the zero magnetic field case (by which $\Phi$ is real), the local coordinate frame is defined by a unit vector along the Knight field $\hat{\mathbf{z}}^{\prime}=\mathbf{K} / K$ :

$$
\hat{\mathbf{z}}^{\prime}=[\sin \phi \operatorname{Re}(a) /|a|, \sin \phi \operatorname{Im}(a) /|a|, \cos \phi] .
$$

Here, $\phi$ is the angle between the original and the rotated $z$ axes, $\cos \phi=\hat{\mathbf{z}} \cdot \hat{\mathbf{z}}^{\prime}=\Phi / \sqrt{3 \Phi^{2} / 4+|a|^{2}}$. We choose the remaining two axes of the rotated coordinate system arbitrarily as

$$
\hat{\mathbf{y}}^{\prime}=[-\operatorname{Im}(a) /|a|, \operatorname{Re}(a) /|a|, 0]
$$

and

$$
\hat{\mathbf{x}}^{\prime}=\hat{\mathbf{y}}^{\prime} \times \hat{\mathbf{z}}^{\prime}=[\cos \phi \operatorname{Re}(a) /|a|, \cos \phi \operatorname{Im}(a) /|a|,-\sin \phi] .
$$

Using the projectors into the transversal plane of the local coordinate system $\mathbf{r}_{ \pm}^{\prime}$, one can evaluate the amplitudes of the 
spin-nonconserving terms. As an auxiliary result, we note that for any real vector $\mathbf{v}$ we have

$$
\mathbf{r}_{ \pm}^{\prime} \cdot \mathbf{v}=v_{+} \frac{a^{*}}{|a|} \frac{\cos \phi \pm 1}{2}+v_{-} \frac{a}{|a|} \frac{\cos \phi \mp 1}{2}-v_{z} \sin \phi .
$$

For example, close to the dot center, the inequality $\Phi \gg|a|$ gives $\sin \phi \approx|a| / \sqrt{3} / 2 \Phi, \cos \phi \approx 1,|a| \sim \lambda_{1} \Phi$, and the Knight field derivative follows as

$$
\mathbf{r}_{ \pm}^{\prime} \cdot \nabla \mathbf{K} \sim \delta^{-1} \sqrt{3} \lambda_{1} \beta\left|\Phi_{00,0}^{\mathrm{hh}}\right|^{2},
$$

where, again, the length $\delta$ depends on the direction of the phonon polarization vector, $\delta=l$ for $\mathbf{e}_{\mathbf{Q}} \perp \hat{\mathbf{z}}$ and $\delta=w / \pi$ for $\mathbf{e}_{\mathbf{Q}} \| \hat{\mathbf{z}}$.

\section{APPENDIX D: INTERACTIONS IN THE IMPURITY ENSEMBLE: VECTORS A AND THE EFFECTIVE HAMILTONIAN TERMS}

The interaction part of the effective Hamiltonian in Eq. (43) for a given impurity pair $n, m$ equals $1 /\left(E_{G}-E_{p}\right)$ times the following expression:

$$
\begin{aligned}
\left(\mathbf{A}^{n} \cdot\right. & \left.\mathbf{I}^{n}\right)\left(\mathbf{A}^{m} \cdot \mathbf{I}^{m}\right)^{\dagger}+\left(\mathbf{A}^{m} \cdot \mathbf{I}^{m}\right)\left(\mathbf{A}^{n} \cdot \mathbf{I}^{n}\right)^{\dagger} \\
= & I_{z}^{n} I_{z}^{m}\left(A_{z}^{n} A_{z}^{m *}+A_{z}^{m} A_{z}^{n *}\right)+I_{+}^{n} I_{-}^{m}\left(A_{-}^{n} A_{-}^{m *}+A_{+}^{m} A_{+}^{n *}\right) / 4 \\
& +I_{-}^{n} I_{+}^{m}\left(A_{+}^{n} A_{+}^{m *}+A_{-}^{m} A_{-}^{n *}\right) / 4+I_{z}^{n} I_{+}^{m}\left(A_{z}^{n} A_{+}^{m *}\right. \\
& \left.+A_{-}^{m} A_{z}^{n *}\right) / 2+I_{+}^{n} I_{z}^{m}\left(A_{-}^{n} A_{z}^{m *}+A_{z}^{m} A_{+}^{n *}\right) / 2 \\
& +I_{z}^{n} I_{-}^{m}\left(A_{z}^{n} A_{-}^{m *}+A_{+}^{m} A_{z}^{n *}\right) / 2+I_{-}^{n} I_{z}^{m}\left(A_{+}^{n} A_{z}^{m *}\right. \\
& \left.+A_{z}^{m} A_{-}^{n *}\right) / 2+I_{+}^{n} I_{+}^{m}\left(A_{-}^{n} A_{+}^{m *}+A_{-}^{m} A_{+}^{n *}\right) / 4 \\
& +I_{-}^{n} I_{-}^{m}\left(A_{+}^{n} A_{-}^{m *}+A_{+}^{m} A_{-}^{n *}\right) / 4 .
\end{aligned}
$$

If the vectors are expressed in the local coordinate frame [that is, all quantities in Eq. (D1) with tildes], the first three terms are spin preserving (connect states with the sum of spin projections along the local spin quantization axes), the next four terms change the sum by one (representing a single spin flip), and the last two terms induce double flips. The complex conjugates are defined as

$$
A_{ \pm}^{m *} \equiv\left(\mathbf{r}_{ \pm}^{\prime} \cdot \mathbf{A}^{m}\right)^{*}=A_{x}^{m *} \mp \mathrm{i} A_{y}^{m *}=\mathbf{r}_{\mp}^{\prime} \cdot\left(\mathbf{A}^{m}\right)^{*} .
$$

To find the effective Hamiltonian, it remains to evaluate the vectors A. For electrons, we assume that the magnetic field dominates the total field for impurities. Equation (25) gives

$$
\begin{aligned}
\left(J=\frac{1}{2}, J^{\prime}=\right. & \left.-\frac{1}{2}\right) \\
& \mathbf{A}^{n}=-(\beta / 2)\left|\Phi_{G}\left(\mathbf{R}_{n}\right)\right|^{2} R_{U_{n}}\left[\mathbf{r}_{-}^{\prime}\right],
\end{aligned}
$$

where we remind that the vectors $\hat{\mathbf{x}}^{\prime}, \hat{\mathbf{y}}^{\prime}$, and $\hat{\mathbf{s}}_{0}=\hat{\mathbf{z}}^{\prime}$ form an orthonormal set, with $\hat{\mathbf{s}}_{0}$ along the external field. Expanding the rotation operator in the lowest order in the spin-orbit length we finally find

$$
A_{-}^{n} \sim-(\beta / 2)\left|\Phi_{G}\left(\mathbf{R}_{n}\right)\right|^{2}, \quad A_{+}^{n}, A_{z}^{n} \sim O\left(r_{n} / l\right) A_{-}^{n},
$$

from where Eq. (50) of the main text follows.

For holes, we find the vectors A corresponding to the interaction mediated by the $-\frac{3}{2}$ state from Eqs. (B5) and (C12) as

$$
\begin{aligned}
\mathbf{r}_{+}^{\prime} \cdot \mathbf{A} & =-\beta b^{2}(a /|a|)(\cos \phi+1), \\
\mathbf{r}_{-}^{\prime} \cdot \mathbf{A} & =-\sqrt{3} \beta \Phi b(a /|a|)(\cos \phi+1), \\
\hat{\mathbf{z}}^{\prime} \cdot \mathbf{A} & =-\sqrt{3} \beta \Phi b(a /|a|) \cos \phi .
\end{aligned}
$$

The largest spin-nonconserving term of the corresponding effective Hamiltonian is

$$
H_{++}^{n m} \sim \tilde{I}_{+}^{n} \tilde{I}_{+}^{m}\left(\frac{1}{E_{G}-E_{p}} 2 \sqrt{3}\left(\beta \Phi^{2}\right)^{2} \lambda_{0}^{2}\right) .
$$

The vectors A for the spin-alike light hole, using Eqs. (B8), follow as

$$
\begin{aligned}
\mathbf{r}_{+}^{\prime} \cdot \mathbf{A} & =-\sqrt{3} \beta \Phi^{*} \Phi^{\prime}\left(a^{*} /|a|\right)(\cos \phi+1) / 2, \\
\mathbf{r}_{-}^{\prime} \cdot \mathbf{A} & =-\beta \Phi^{\prime} b^{*}(\cos \phi+1), \\
\hat{\mathbf{z}}^{\prime} \cdot \mathbf{A} & =-(\beta / 2)\left(3 a^{\prime} \Phi^{*}+a^{*} \Phi^{\prime}\right) \cos \phi .
\end{aligned}
$$

From the above results, we see that the largest spinnonconserving terms in the effective interaction are

$$
H_{++}^{n m} \sim \tilde{I}_{+}^{n} \tilde{I}_{+}^{m}\left(\frac{1}{\Delta_{\mathrm{lh}}} \sqrt{3}\left(\beta \Phi^{2}\right)^{2} \lambda_{0}\right)
$$

and

$$
H_{+z}^{n m} \sim \tilde{I}_{+}^{n} \tilde{I}_{z}^{m}\left(\frac{1}{\Delta_{\mathrm{lh}}}(3 / 4) \sqrt{3}\left(\beta \Phi^{2}\right)^{2} \lambda_{1}^{\prime}\right) .
$$

Similarly as before, a differentiation with respect to the position, which enters the relaxation rates, can be in Eqs. (D6)-(D9) approximated as a multiplication by $1 / \delta$.

\section{APPENDIX E: MATERIALS PARAMETERS}

For the electronic case, we assume a GaAs/AlGaAs heterostructure with the following parameters: electron effective

TABLE II. $g$ factor, magnetic moment, spin, the corresponding energy scale, and the effective field $B_{\text {eff }}$. For GaAs nuclear spins, the $g$ factor is the average over naturally abundant isotopes. The effective field for impurities is the Knight field $B_{\text {eff }}=J(\beta / V)$, for the particle it is the Overhauser field $B_{\text {eff }}=\sqrt{N I(I+1)}(\beta / V)$.

\begin{tabular}{lcccc}
\hline \hline Quantity & Nucleus & Mn & Electron & Hole \\
\hline$g$ & 1.2 & 2 & -0.44 & $-\frac{2}{3}$ \\
$\mu$ & $\mu_{N}$ & $\mu_{B}$ & $\mu_{B}$ & $\frac{3}{2}$ \\
$I$ or $J$ & $\frac{3}{2}$ & $\frac{5}{2}$ & $12.7 \mu \mathrm{eV} / \mathrm{T}$ & $58 \mu \mathrm{eV} / \mathrm{T}$ \\
$g \mu I$ & $57 \mathrm{neV} / \mathrm{T}$ & $290 \mu \mathrm{eV} / \mathrm{T}$ & $670 \mathrm{mK} / \mathrm{T}$ \\
$g \mu I / k_{B}$ & $660 \mu \mathrm{K} / \mathrm{T}$ & $3.4 \mathrm{~K} / \mathrm{T}$ & $146 \mathrm{mK} / \mathrm{T}$ & $13.8 x_{\mathrm{Mn}}^{1 / 2} \mathrm{meV}$ \\
$B_{\text {eff }}$ & $33 \mathrm{peV}$ & $96 \mu \mathrm{eV}$ & $145 \mathrm{neV}$ & $237 x_{\mathrm{Mn}}^{1 / 2} \mathrm{~T}$ \\
$B_{\text {eff }} /(g \mu I)$ & $0.6 \mathrm{mT}$ & $331 \mathrm{mT}$ & $12 \mathrm{mT}$ & \\
\hline \hline
\end{tabular}


TABLE III. Effective volume, number of impurities, and the coupling energy scale.

\begin{tabular}{lcc}
\hline \hline Quantity & Electron & Hole \\
\hline$V$ & $3 \times 10^{4} \mathrm{~nm}^{3}$ & $238 \mathrm{~nm}^{3}$ \\
$N$ & $1.3 \times 10^{6}$ & $x_{\mathrm{Mn}} \times 5278$ \\
$\beta / V$ & $66 \mathrm{peV}$ & $64 \mu \mathrm{eV}$ \\
\hline \hline
\end{tabular}

mass $m=0.067 m_{0}$, in-plane confinement length $l=30 \mathrm{~nm}$, quantum well width $w=8 \mathrm{~nm}$, spin-orbit length $l_{\mathrm{SO}} \sim 1 \mu \mathrm{m}$, electron-nuclear coupling $\beta=2 \mu \mathrm{eV} \mathrm{nm}{ }^{3}$, material density $\rho=5300 \mathrm{~kg} / \mathrm{m}^{3}$, phonon velocities $c_{l}=5290 \mathrm{~m} / \mathrm{s}$, and $c_{t}=2480 \mathrm{~m} / \mathrm{s}$, conduction band piezoelectric $\Xi=1.4 \times$ $10^{9} \mathrm{eV} / \mathrm{m}$, and deformation $\sigma=10 \mathrm{eV}$ potentials. The $g$ factors and corresponding energy scales are given in Table II.

For the hole case, we list the Luttinger parameters $\gamma_{1} / \gamma_{2} / \gamma_{3}$ of GaAs (Refs. 104 and 105): 7.1/2/2.9, CdTe (Ref. 106): 4.1/1.1/1.6, and ZnTe (Ref. 107): 3.8/0.7/1.3. We take ZnTe as the material of our choice, with $\rho=5650 \mathrm{~kg} / \mathrm{m}^{3}, c_{l}=$ $3550 \mathrm{~m} / \mathrm{s}, c_{t}=2358 \mathrm{~m} / \mathrm{s}, \sigma=5 \mathrm{eV}, \Xi=3.4 \times 10^{8} \mathrm{eV} / \mathrm{m}$. We set the heavy-hole orbital energy $\hbar \Omega_{\mathrm{hh}}$ to $20 \mathrm{meV}$, which gives $l_{\mathrm{hh}}=4.19 \mathrm{~nm}, l_{\mathrm{lh}}=3.82 \mathrm{~nm}$, and $\hbar \Omega_{\mathrm{lh}}=17 \mathrm{meV}$. We set the light-heavy-hole splitting $\Delta_{\mathrm{lh}}$ to $100 \mathrm{meV}$, which gives $w=3.24 \mathrm{~nm}$. The hole-impurity interaction strength is $\beta=$ $1 / 3 \mathrm{eV} a_{0}^{3} / 4$, with $a_{0}=0.61 \mathrm{~nm}$ the lattice constant. We assume the Mn impurities concentration is given as $x_{\mathrm{Mn}}$, the ratio of cation replaced by $\mathrm{Mn}$ atoms, typically of the order of $1 \%$.

Properly normalized, the ground state follows from Eq. (A7) as

$$
\Phi_{00,0}^{J}(r, \phi, z)=\sqrt{\frac{2}{w}} \sin \left(\frac{\pi z}{w}\right) \frac{1}{\sqrt{\pi} l_{J}} \exp \left(-\frac{r^{2}}{2 l_{J}^{2}}\right),
$$

from where the quantum dot volume estimate

$$
V=1 / \int d^{3} \mathbf{R}|\Phi(\mathbf{R})|^{4}=(4 \pi / 3) w l_{J}^{2}
$$

gives values in Table III.

From the above parameters, the hole admixture coefficients follow as $\lambda_{0} \approx 0.053, \lambda_{1} \approx 0.050, \lambda_{0}^{\prime} \approx 0.11$, and $\lambda_{1}^{\prime} \approx 0.15$.

\section{APPENDIX F: ALTERNATIVE HOLE-MIXING MECHANISMS}

In this section, we estimate two additional light-heavyhole admixture sources, namely, the spin-orbit coupling to an external electric field (the Rashba spin-orbit interaction) and Overhauser impurity field itself. We quantify the resulting light-heavy-hole admixture by calculating the corresponding coefficients $\lambda$. We find that these alternatives lead to a smaller amount of admixture, compared to the terms of the KohnLuttinger Hamiltonian we considered in the main text.

An electric field $\mathbf{E}$ induces the following term in the hole Hamiltonian (in the notation of Ref. 100):

$$
H_{8 v 8 v}^{r}=r_{41}^{8 v 8 v} \mathbf{E} \cdot \mathbf{J} \times \mathbf{k} .
$$

Assuming, for convenience, that the field is perpendicular to the heterostructure interface $\mathbf{E}=\hat{\mathbf{z}} E$, this term translates into our notation of Appendix A as

$$
H_{ \pm 3 / 2, \pm 1 / 2}^{\mathrm{SO}}= \pm r_{41}^{8 v 8 v} E \sqrt{3} k_{\mp} / 2,
$$

where the material parameter $r_{41}^{8 v 8 v}$ we estimate by its $\mathrm{ZnSe}$ value of $-4.1 \mathrm{e}^{2}$. Taking Eq. (A7) for the heavy-hole ground state $\left|\Psi_{3 / 2}\right\rangle$, the Rashba spin-orbit interaction leads to an admixture of the light-hole state $|1 / 2\rangle \otimes\left|\Phi_{01,0}^{\mathrm{lh}}\right\rangle$ with a coefficient

$$
\lambda_{\mathrm{SO}}=-\frac{\mathrm{i}}{2} \frac{\sqrt{3} r_{41}^{8 v 8 v} E}{\Delta_{\mathrm{lh}}+\hbar \Omega_{\mathrm{lh}}} .
$$

To quantify its value, it remains to put in the value for the electric field $E$. We estimate it to be of order $10^{7} \mathrm{~V} / \mathrm{m}$, using a crude electrostatic model; namely, we take the hole to be a point charge in the center of a half-sphere of a radius $l \sim 5 \mathrm{~nm}$ and the electron to be a uniform classical charge density $\sigma$ on the half-sphere, which corresponds to the type-II quantum dot populated by a single exciton. The resulting internal field $E=$ $\sigma / 2 \epsilon$ is smaller than the piezoelectric field accompanying the phonon [Eq. (78)], which we estimated to be of order $10^{8} \mathrm{~V} / \mathrm{m}$. Inserting this latter value for $E$, we find $\lambda^{\mathrm{SO}} \approx \sqrt{ } 3 \times 10^{-3}$, so that both the internal electric field as well as phonon-induced fields induce light-heavy-hole admixtures much smaller than those we considered in the main text.

One may also consider the collective field of the impurities (the Overhauser field) as a source of the light-heavy-hole mixing. Using Eqs. (B8), we estimate the arising admixture of the light-hole state $|1 / 2\rangle \otimes\left|\Phi_{00,0}^{\mathrm{lh}}\right\rangle$ into the heavy-hole ground state

$$
\left|\lambda_{\mathrm{Mn}}\right|^{2} \approx \frac{(\beta / V)^{2} N I(I+1)}{2 \Delta_{\mathrm{lh}}} .
$$

The previous is a typical value, the phase being fixed by the microscopic state of the Mn ensemble. Evaluating for our parameters, we get $\lambda_{\mathrm{Mn}} \approx 7 \times 10^{-3}$, typically an order of magnitude smaller admixture as that considered in the main text, so we can again neglect this admixture source.
${ }^{1}$ D. Loss and D. P. DiVincenzo, Phys. Rev. A 57, 120 (1998).

${ }^{2}$ R. Hanson, L. P. Kouwenhoven, J. R. Petta, S. Tarucha, and L. M. K. Vandersypen, Rev. Mod. Phys. 79, 1217 (2007).

${ }^{3}$ J. M. Taylor, J. R. Petta, A. C. Johnson, A. Yacoby, C. M. Marcus, and M. D. Lukin, Phys. Rev. B 76, 035315 (2007).

${ }^{4}$ F. H. L. Koppens, C. Buizert, K. J. Tielrooij, I. T. Vink, K. C. Nowack, T. Meunier, L. P. Kouwenhoven, and L. M. K. Vandersypen, Nature (London) 442, 766 (2006).

${ }^{5}$ K. C. Nowack, F. H. L. Koppens, Y. V. Nazarov, and L. M. K. Vandersypen, Science 318, 1430 (2007).
${ }^{6}$ T. Obata, M. Pioro-Ladriere, Y. Tokura, Y.-S. Shin, T. Kubo, K. Yoshida, T. Taniyama, and S. Tarucha, Phys. Rev. B 81, 085317 (2010).

${ }^{7}$ D. Press, T. D. Ladd, B. Zhang, and Y. Yamamoto, Nature (London) 456, 218 (2008)

${ }^{8}$ J. Fabian, A. Matos-Abiague, C. Ertler, P. Stano, and I. Žutić, Acta Phys. Slov. 57, 565 (2007); arXiv:0711.1461.

${ }^{9}$ I. Žutić, J. Fabian, and S. Das Sarma, Rev. Mod. Phys. 76, 323 (2004).

${ }^{10}$ W. A. Coish and J. Baugh, Phys. Status Solidi B 246, 2203 (2009). 
${ }^{11}$ S. I. Erlingsson, Y. V. Nazarov, and V. I. Falko, Phys. Rev. B 64, 195306 (2001).

${ }^{12}$ A. V. Khaetskii, D. Loss, and L. Glazman, Phys. Rev. Lett. 88, 186802 (2002).

${ }^{13}$ I. A. Merkulov, A. L. Efros, and M. Rosen, Phys. Rev. B 65, 205309 (2002).

${ }^{14}$ W. A. Coish, V. N. Golovach, and J. C. Egues, Phys. Status Solidi B 243, 3658 (2006).

${ }^{15}$ L. Cywinski, W. M. Witzel, and S. Das Sarma, Phys. Rev. B 79, 245314 (2009).

${ }^{16}$ L. Cywinski, W. M. Witzel, and S. Das Sarma, Phys. Rev. Lett. 102, 057601 (2009).

${ }^{17}$ I. A. Merkulov, G. Alvarez, D. R. Yakovlev, and T. C. Schulthess, Phys. Rev. B 81, 115107 (2010).

${ }^{18}$ J. R. Petta, A. C. Johnson, J. M. Taylor, E. A. Laird, A. Yacoby, M. D. Lukin, C. M. Marcus, M. P. Hanson, and A. C. Gossard, Science 309, 2180 (2005).

${ }^{19}$ F. H. L. Koppens, K. C. Nowack, and L. M. K. Vandersypen, Phys. Rev. Lett. 100, 236802 (2008).

${ }^{20}$ H. Bluhm, S. Foletti, I. Neder, M. Rudner, D. Mahalu, V. Umansky, and A. Yacoby, Nat. Phys. 7, 109 (2010).

${ }^{21}$ J. Schliemann, A. Khaetskii, and D. Loss, J. Phys.: Condens. Matter 15, R1809 (2003).

${ }^{22}$ D. J. Reilly, J. M. Taylor, J. R. Petta, C. M. Marcus, M. P. Hanson, and A. C. Gossard, Science 321, 5890 (2008).

${ }^{23}$ A. S. Bracker, E. A. Stinaff, D. Gammon, M. E. Ware, J. G. Tischler, A. Shabaev, A. L. Efros, D. Park, D. Gershoni, V. L. Korenev et al., Phys. Rev. Lett. 94, 047402 (2005).

${ }^{24}$ C. W. Lai, P. Maletinsky, A. Badolato, and A. Imamoglu, Phys. Rev. Lett. 96, 167403 (2006).

${ }^{25}$ A. Hgele, M. Kroner, C. Latta, M. Claassen, I. Carusotto, C. Bulutay, and A. Imamoglu, Phys. Rev. Lett. 108, 197403 (2012).

${ }^{26}$ J. Seufert, G. Bacher, M. Scheibner, A. Forchel, S. Lee, M. Dobrowolska, and J. K. Furdyna, Phys. Rev. Lett. 88, 027402 (2001).

${ }^{27}$ L. Besombes, Y. Léger, L. Maingault, D. Ferrand, H. Mariette, and J. Cibert, Phys. Rev. Lett. 93, 207403 (2004).

${ }^{28}$ F. Xiu, Y. Wang, J. Kim, Y. Zhou, X. Kou, W. Han, R. K. Kawakami, J. Zou, and K. L. Wang, ACS Nano. 4, 4948 (2010).

${ }^{29}$ L. Klopotowski, L. Cywiński, P. Wojnar, V. Voliotis, K. Fronc, T. Kazimierczuk, A. Golnik, M. Ravaro, R. Grousson, G. Karczewski et al., Phys. Rev. B 83, 081306(R) (2011).

${ }^{30}$ A. A. Maksimov, G. Bacher, A. McDonald, V. D. Kulakovskii, A. Forchel, C. R. Becker, G. Landwehr, and L. W. Molenkamp, Phys. Rev. B 62, R7767 (2000).

${ }^{31}$ P. S. Dorozhkin, A. V. Chernenko, V. D. Kulakovskii, A. S. Brichkin, A. A. Maksimov, H. Schoemig, G. Bacher, A. Forchel, S. Lee, M. Dobrowolska et al., Phys. Rev. B 68, 195313 (2003).

${ }^{32}$ H. J. Meng, J. Lu, P. H. T. S. Yan, and J. H. Zhao, Europhys. Lett. 84, 58007 (2008).

${ }^{33}$ R. Beaulac, L. Schneider, P. I. Archer, G. Bacher, and D. R. Gamelin, Science 325, 973 (2009).

${ }^{34}$ I. R. Sellers, R. Oszwałdowski, V. R. Whiteside, M. Eginligil, A. Petrou, I. Žutić, W.-C. Chou, W. C. Fan, A. G. Petukhov, S. J. Kim et al., Phys. Rev. B 82, 195320 (2010).

${ }^{35}$ A. O. Govorov, Phys. Rev. B 72, 075359 (2005).

${ }^{36}$ A. O. Govorov, C. R. Phys. 9, 857 (2008).

${ }^{37}$ J. Fernández-Rossier and L. Brey, Phys. Rev. Lett. 93, 117201 (2004).
${ }^{38}$ F. Qu and P. Hawrylak, Phys. Rev. Lett. 96, 157201 (2006).

${ }^{39}$ N. T. T. Nguyen and F. M. Peeters, Phys. Rev. B 78, 045321 (2008).

${ }^{40}$ R. M. Abolfath, P. Hawrylak, and I. Žutić, Phys. Rev. Lett. 98, 207203 (2007).

${ }^{41}$ R. Oszwałdowski, I. Žutić, and A. G. Petukhov, Phys. Rev. Lett. 106, 177201 (2011).

${ }^{42}$ N. Lebedeva, A. Varpula, S. Novikov, and P. Kuivalainen, Phys. Rev. B 81, 235307 (2010).

${ }^{43}$ D. R. Yakovlev and W. Ossau, in Introduction to the Physics of Diluted Magnetic Semiconductors, edited by J. Kossut and J. A. Gaj (Springer, Berlin, 2010).

${ }^{44}$ T. Dietl and J. Spalek, Phys. Rev. B 28, 1548 (1983).

${ }^{45}$ P. A. Wolff, in Semiconductors and Semimetals, edited by J. K. Furdyna and J. Kossut, Vol. 25 (Academic, San Diego, 1988).

${ }^{46}$ E. L. Nagaev, Physics of Magnetic Semiconductors (MIR, Moscow, 1983).

${ }^{47}$ J. K. Furdyna, J. Appl. Phys. 64, R29 (1988).

${ }^{48}$ S. Amasha, K. MacLean, I. P. Radu, D. M. Zumbühl, M. A. Kastner, M. P. Hanson, and A. C. Gossard, Phys. Rev. Lett. 100, 046803 (2008).

${ }^{49}$ A. V. Akimov, A. V. Scherbakov, D. R. Yakovlev, I. A. Merkulov, M. Bayer, A. Waag, and L. W. Molenkamp, Phys. Rev. B 73, 165328 (2006).

${ }^{50}$ J. J. Baumberg, S. A. Crooker, D. D. Awschalom, N. Samarth, H. Luo, and J. K. Furdyna, Phys. Rev. B 50, 7689 (1994).

${ }^{51}$ E. Tsitsishvili and H. Kalt, Phys. Rev. B 77, 155305 (2008).

${ }^{52}$ D. Paget, G. Lampel, B. Sapoval, and V. I. Safarov, Phys. Rev. B 15, 5780 (1977)

${ }^{53}$ M. C. Kuo, J. S. Hsu, J. L. Shen, K. C. Chiu, W. C. Fan, Y. C. Lin, C. H. Chia, W. C. Chou, M. Yasar, R. Mallory et al., Appl. Phys. Lett. 89, 263111 (2006).

${ }^{54}$ This is further corroborated by applying ZnTe parameters to the theoretical approach from Ref. 108.

${ }^{55} \mathrm{R}$. de Sousa, in Electron Spin Resonance and Related Phenomena in Low-Dimensional Structures, Topics in Applied Physics, edited by M. Fianculli, Vol. 115 (Sanremo, Italy, 2009), p. 183.

${ }^{56}$ T. Dietl, P. Peyla, W. Grieshaber, and M. dÁubign, J. Magn. Magn. Mater. 140-144, 2051 (1995).

${ }^{57}$ A. Hundt, J. Puls, A. V. Akimov, Y. H. Fan, and F. Henneberger, Phys. Rev. B 72, 033304 (2005).

${ }^{58}$ M. K. Kneip, D. R. Yakovlev, M. Bayer, A. A. Maksimov, I. I. Tartakovskii, D. Keller, W. Ossau, L. W. Molenkamp, and A. Waag, Phys. Rev. B 73, 035306 (2006).

${ }^{59}$ J. M. Elzerman, R. Hanson, L. H. W. van Beveren, B. Witkamp, L. M. K. Vandersypen, and L. P. Kouwenhoven, Nature (London) 430, 431 (2004).

${ }^{60}$ I. L. Aleiner and V. I. Faľko, Phys. Rev. Lett. 87, 256801 (2001).

${ }^{61}$ L. S. Levitov and E. I. Rashba, Phys. Rev. B 67, 115324 (2003).

${ }^{62}$ D. Stepanenko, N. E. Bonesteel, D. P. DiVincenzo, G. Burkard, and D. Loss, Phys. Rev. B 68, 115306 (2003).

${ }^{63}$ J. Schliemann, J. C. Egues, and D. Loss, Phys. Rev. Lett. 90, 146801 (2003).

${ }^{64}$ I. V. Tokatly and E. Y. Sherman, Ann. Phys. (NY) 325, 1104 (2010).

${ }^{65}$ I. V. Tokatly and E. Y. Sherman, Phys. Rev. B 82, 161305 (2010).

${ }^{66}$ P. Stano and J. Fabian, Phys. Rev. Lett. 96, 186602 (2006).

${ }^{67}$ F. Baruffa, P. Stano, and J. Fabian, Phys. Rev. Lett. 104, 126401 (2010).

${ }^{68}$ R. Oswaldowski, P. Stano, A. G. Petukhov, and I. Žutić, Phys. Rev. B 86, 201408(R) (2012). 
${ }^{69}$ J. Fischer, W. A. Coish, D. V. Bulaev, and D. Loss, Phys. Rev. B 78, 155329 (2008).

${ }^{70}$ A. Pfund, I. Shorubalko, K. Ensslin, and R. Leturcq, Phys. Rev. Lett. 99, 036801 (2007).

${ }^{71}$ M. S. Rudner and L. S. Levitov, Phys. Rev. Lett. 99, 036602 (2007).

${ }^{72}$ S. I. Erlingsson and Y. V. Nazarov, Phys. Rev. B 66, 155327 (2002).

${ }^{73}$ N. Shenvi, R. de Sousa, and K. B. Whaley, Phys. Rev. B 71, 224411 (2005).

${ }^{74}$ W. Yao, R.-B. Liu, and L. J. Sham, Phys. Rev. B 74, 195301 (2006).

${ }^{75}$ P. Löwdin, J. Chem. Phys. 19, 1396 (1951).

${ }^{76}$ G. L. Bir and G. E. Pikus, Symmetry and Strain-induced Effects in Semiconductors (Wiley, New York, 1974).

${ }^{77}$ A. Abragam, The Principles of Nuclear Magnetism (Oxford University Press, Oxford, UK, 1961).

${ }^{78}$ While less is known for quantum dots, some guidance about different stages of magnetic polaron formation and the corresponding time scales can be inferred from quantum wells, discussed in Ref. 43, p. 258.

${ }^{79}$ J. Cibert and D. Scalbert, in Diluted Magnetic Semiconductors: Basic Physics and Optical Properties, Spin Physics in Semiconductors, edited by M. I. Dyakonov, Vol. 157 (Springer, Berlin, 2008), pp. 416-417.

${ }^{80}$ B. Braunecker, P. Simon, and D. Loss, Phys. Rev. Lett. 102, 116403 (2009).

${ }^{81}$ J. M. Pientka, R. Oszwałdowski, A. G. Petukhov, J. E. Han, and I. Žutić, Phys. Rev. B 86, 161403(R) (2012).

${ }^{82}$ L. Esaki, P. J. Stiles, and S. von Molnar, Phys. Rev. Lett. 19, 852 (1967).

${ }^{83}$ J. S. Moodera, X. Hao, G. A. Gibson, and R. Meservey, Phys. Rev. Lett. 61, 637 (1988).

${ }^{84}$ J. C. Egues, Phys. Rev. Lett. 80, 4578 (1998).

${ }^{85}$ I. Žutić, J. Fabian, and S. D. Sarma, Appl. Phys. Lett. 82, 221 (2003).

${ }^{86}$ D. P. DiVincenzo, J. Appl. Phys. 85, 4975 (1999).

${ }^{87}$ R. M. Abolfath, A. G. Petukhov, and I. Žutić, Phys. Rev. Lett. 101, 207202 (2008).

${ }^{88}$ D. G. Austing, S. Sasaki, S. Tarucha, S. M. Reimann, M. Koskinen, and M. Manninen, Phys. Rev. B 60, 11514 (1999).
${ }^{89} \mathrm{~F}$. Henneberger and J. Puls, in Introduction to the Physics of Diluted Magnetic Semiconductors, edited by J. Kossut and J. A. Gaj (Springer, Berlin, 2010).

${ }^{90}$ D. R. Yakovlev and I. A. Merkulov, in Introduction to the Physics of Diluted Magnetic Semiconductors, edited by J. Kossut and J. A. Gaj (Springer, Berlin, 2010).

${ }^{91}$ V. I. Klimov, Annu. Rev. Phys. Chem. 58, 635 (2007).

${ }^{92}$ G. D. Scholes, Adv. Funct. Mater. 18, 1157 (2007).

${ }^{93}$ N. P. Stern, M. Poggio, M. H. Bartl, E. L. Hu, G. D. Stucky, and D. D. Awschalom, Phys. Rev. B 72, 161303 (2005).

${ }^{94}$ S. T. Ochsenbein and D. R. Gamelin, Nat. Nanotechnol. 6, 112 (2011).

${ }^{95}$ R. Beaulac, P. I. Archer, S. T. Ochsenbein, and D. R. Gamelin, Adv. Funct. Mater. 18, 3873 (2008).

${ }^{96}$ D. A. Bussian, S. A. Crooker, M. Yin, M. Brynda, A. L. Efros, and V. I. Klimov, Nat. Mater. 8, 35 (2009).

${ }^{97}$ S. T. Ochsenbein, Y. Feng, K. M. Whitaker, E. Badaeva, W. K. Liu, X. Li, and D. R. Gamelin, Nat. Nanotechnol. 4, 681 (2009).

${ }^{98}$ I. Žutić and A. G. Petukhov, Nat. Nanotechnol. 4, 623 (2009).

${ }^{99}$ R. Viswanatha, J. M. Pietryga, Victor I. Klimov, and S. A. Crooker, Phys. Rev. Lett. 107, 067402 (2011).

${ }^{100} \mathrm{R}$. Winkler, Spin-Orbit Coupling Effects in Two-Dimensional Electron and Hole Systems (Springer, Berlin, 2003).

${ }^{101}$ A. K. Bhattacharjee, Phys. Rev. B 76, 075305 (2007).

${ }^{102}$ J. Fischer and D. Loss, Phys. Rev. Lett. 105, 266603 (2010).

${ }^{103}$ G. Katsaros, V. N. Golovach, P. Spathis, N. Ares, M. Stoffel, F. Fournel, O. G. Schmidt, L. I. Glazman, and S. De Franceschi, Phys. Rev. Lett. 107, 246601 (2011).

${ }^{104}$ N. Binggeli and A. Baldereschi, Phys. Rev. B 43, 14734 (1991).

${ }^{105}$ B. V. Shanabrook, O. J. Glembocki, D. A. Broido, and W. I. Wang, Phys. Rev. B 39, 3411 (1989).

${ }^{106}$ T. Friedrich, J. Kraus, G. Schaack, and W. O. G. Schmitt, J. Phys.: Condens. Matter 6, 4307 (1994).

${ }^{107}$ H. Wagner, S. Lankes, K. Wolf, W. Kuhn, P. Link, and W. Gebhardt, J. Cryst. Growth 117, 303 (1992).

${ }^{108}$ K. Vyborny, J. E. Han, R. Oszwałdowski, I. Žutić, and A. G. Petukhov, Phys. Rev. B 85, 155312 (2012). 